\title{
Stock Market Seasonality- Time Varying Volatility In The Emerging Indian Stock Market
}

\author{
${ }^{1}$ Dr.A.Arumugam, ${ }^{2 \cdot}$ Dr.K.Soundararajan \\ 1. Professor, Dept. of Management Studies Paavai Engineering College,Namakkal-637018.Tamil \\ Nadu,India. \\ 2.Professor, Dept. of Management Studies Dhanalakshmi Seenivasan Engineering College, Perambalur, Tamil \\ Nadu, India
}

\begin{abstract}
This paper investigates seasonality and time varying volatility in the Indian stock markets. The researcher finds that there is a divergent cyclic pattern in investor actions that is reflecting not only returns, but in all aspects of trade activity. The information diffusion apparatus ensures that the stock returns across all days of the weeks and months are equal and the market participant, the balanced financial decision-maker, cannot earn any extra-normal profits. It is important to note that there are variations in explosive nature of stock takings by the "each day-of-the Week", "every month of the Year" and "Semi-Month". Besides, a high (low) return is connected with a correspondingly high (low) volatility for a given day. If the investors can recognize a certain pattern in instability, then it would be easier to make investment decision based on both returns and risk. Studies on the changes in the market return and stock return of companies facilitate the government to know whether the objectives of the monetary system have been realized or not. The result of the analysis enables the establishment to reformulate the system wherever required. In a developing country like India, fast development of economy is very important. It can be realized by having trade development. For which, the stock market contributes more for the development of industries in general and economic development in particular. Unless periodical assessment made, the extent of achieving this object will not be known. In a vibrant economic system periodical review is much more important. It helps to review and readjust the policy according to change of time. Changes in the market return and stock return of the selected companies have been analysed on daily basis, monthly basis and yearly basis. The present study also premeditated the risk parameter for the market return and stock return of the selected companies.
\end{abstract}

Keywords: Seasonality; Returns, volatility and volume traded; Calendar (Day, Week and Month) effects; Indian stock markets; Window dressing , Earning season, Efficient market hypothesis and Festival holiday.

\section{Introduction}

The ripples generate by affairs of state and macroeconomic announcements may tricky to understand broader patterns in the stock market. These patterns may replicate on a seasonal basis, or occur more at asymmetrical intervals. Seasonal patterns are anchor in yearly taxation milestone, pension and bonus payments, the quarterly "earnings season", "window-dressing" on the part of fund managers, index-rebalancing periods, possible seasonal psychological effects, production, advertising, and release cycles in industry, and a likely innumerable of other factors that are more complicated to identify and isolate. Apparently, one should not expect seasonal patterns to be set in stone as tax rates and schedules change, so should the joined seasonal effects. Despite something of a disagreement of the "efficient market hypothesis", though, these patterns do tend to keep it up persist and can be taken advantage of...most of the seasonal investment tests to the right of the screen have outperformed the general market is very radically on scales as small as a day to as long as 3 months. The values that underlies the seasonal investing plan is that more losses can be eliminated or abridged, the greater is the fall of risk and therefore the better the chance for greater profits.

For this research purposes, the word "seasonality" is defined as a number of cyclic trends that seem to exist in the stock market. A seasonal movement is a relentless time period when the stock market has a statistically high predisposition to either rise or fall. Seasonal investing relies on probabilities, uncertainties, and it underperforms due to conventional investment methods.

\section{Objectives of the Study}

The main objectives of the research is to know the instability of market return and stock return of the sample companies listed at the Bombay Stock Exchange and at the National Stock Exchange and the seasonality of the Indian stock market with reference to time varying unpredictability. . 


\section{Literature review}

Hansen, Charlotte Strunk (This work has got its own methodology. The study uses only secondary data for analytical purpose. Secondary data are not originally collected rather obtained from published or unpublished sources. The data for the present study were collected from the websites of the Bombay Stock Exchange and from the National Stock Exchange. The data also collected from the "money" website. The collected raw data were classified and computed according to requirement of the study. With a view to study the volatility and seasonality of the Indian stock market the appropriate statistical tools have been used. This analysis further has been interpreted and observation is made out of it.

A brief review of select studies has been presented here to identify research gap and understand methodologies employed in the research area of Seasonality and volatility of Indian stock market 2000) This paper examines the information content of options on the Danish KFX share index. It considered the relation between the volatility implied in an option's price and the subsequently realised index return volatility. Since these options are traded infrequently and in low volumes, the errors-in-variables problem is potentially large. They address the problem directly using instrumental variables techniques. It was fond that when measurement errors are controlled for, call option prices even in this very illiquid market contains information about future realised volatility over and above the information contained in historical volatility. ${ }^{1}$

T Lavin, Angeline's (2000) found that evidence of seasonality in stock returns during the 1926-92 periods. Dividing the data into sub-periods yields the following results: there was no evidence of stock market seasonality from 1926 to 1940, seasonality increased between 1941 and 1975 and then diminished slightly from 1976 to 1992 . Specifically, the average January return was found to be significantly different than the average return in the other eleven months of the year. Seasonality was found in the high-quality end of the corporate bond market during the 1966-78 periods, but there was no evidence of seasonality in the government bond market. $^{2}$

Amanulla.S and Thiripalraju (2001), found that there was consistent positive returns on Wednesdays and negative returns on Tuesdays due to possible impact of the Week End Effect. ${ }^{3}$

Moses, Lucia (2001) opined that Veronis' report forecasts newspaper ad spending will grow at a compound annual rate of $5.7 \%$ the next five years. But with no new advertising streams on the horizon to replace the tech and Internet ads that drove spending in the late 1990s, Veronis predicts media properties won't recover as quickly as they did from the last recession.After years of publishers curtailing outlying circulation and shuttering evening editions, Broadwater said he is glad to see publishers' recent commitment to turning around circulation declines, a move crucial to their survival. ${ }^{4}$

Unsworth, Edwin (2002) concluded that European insurers and reinsurers, which traditionally have placed more of their investments in equities than their U.S. counterparts, recently began announcing half-year results that illustrate the adverse effects of lower investment earnings on profits. These companies, their customers and insurer rating agencies are all becoming concerned about the consequences of lower investment returns and losses.Until recently, insurers and reinsurers in continental Europe have placed between $20 \%$ and $25 \%$ of their investments in equities, though that percentage was as high as $80 \%$ for U.K. life insurance companies, according to Yann Le Pallec, a Paris-based analyst with Standard \& Poor's Corp. This compares with the 1960s and 1970s, when insurer investment practices were "very conservative" with about $80 \%$ of assets invested in government bonds and most of the remainder in real estate rather than equities. ${ }^{5}$

.A study of the U.S. (2002), equity market and the indices that track it will use a measure I call the "share-weighted return." This particular figure is not provided by Morningstar or other services. The shareweighted return assigns a greater weight to stocks that have more shares outstanding and less weight to thinlyheld stocks. As a result, this procedure attempts to track equity returns in proportion to the share base held by investors. Like market cap weighting, the technique acknowledges the inherent size and scope differentials among U.S. equities. But unlike market cap weighting, it does not use current stock prices in the calculation. Shares outstanding and their quarterly or annual returns are the components. ${ }^{6}$

Harper, Richard B., (2003) stated that investors hold cash and near-cash balances for several reasons. One typical reason to hold demand deposits and money market fund balances is to provide ready access to cash for various transaction needs and unanticipated liquidity needs. Many investors also hold cash and near-cash balances to moderate their risk exposure to equity markets and provide peace of mind and shelter from the storm when stock prices seem dangerously high or when stock market volatility soars.Fixed principal accounts are less appropriate in asset allocation and balanced account applications, though, and cash-equivalents can be a detriment to the performance of a balanced portfolio in terms of both return and downside risk protection. ${ }^{7}$

Chotigcat .T and Pandey I M. (2005) investigated the Monthly Effect on stock returns for the stock market in India and Malaysia. This study empirically confirmed the existence of Seasonality in stock returns in both capital markets. The study suggested that the Indian Stock Market would move in the direction of higher level of efficiency and the investors would earn returns commensurate with risk. ${ }^{8}$ 
Hareesh Kumar.V and Malabika Deo (2007) analyzed the efficiency of Indian Stock Market by using S\&P CNX 500 Index. They discovered the presence of Day of the Week Effect in the Indian Stock Market, which affected both the stock returns and volatility, thereby proving the Indian Stock Market to be inefficient. ${ }^{9}$

Stovall, Sam(2007) stated that The S\&P 500 index just eclipsed the 1,500 mark for the first time since September 11, 2000. Market sages are now talking about overtaking the S\&P 500's old high of 1527 in the next two months. At this rate, the S\&P 500 could be up $17 \%$ for the full year. But in light of eroding fundamentals, many investors must be wondering if the recent market advance is sustainable. It's no wonder investors are considering the old Wall Street adage: "Sell in May then walk away." S\&P Equity Strategy believes investors would be wise to heed the advice, but in a slightly altered fashion. There is truth in the old adage, in our opinion. Since 1945, the S\&P 500 posted an average price gain of $7.1 \%$ during the November through April [N-A] period, versus a rise of only $1.6 \%$ from May through October [M-O], implying that greater profits could be made elsewhere. In addition, the performance during N-A outperformed M-O 69\% of the time, as was the case in the last 12 months. ${ }^{10}$

Halperin, Alex (2007) revealed that some market pros remain optimistic about Chinese stocks, despite interest rate-hike jitters and periodic bouts of volatility Any lingering doubts about China's global clout were laid to rest when fears that the government would have to raise interest rates caused a brief panic on Apr. 19 in Asian and European markets. News that China's economy had grown at an annualized rate of $11.1 \%$ surpassed expectations, and investors feared China would have to hike rates to stave off inflation and rein in overheated growth. But analysts remain sanguine despite the Middle Kingdom's periodic bouts of volatility, good news for investors in Europe and the U.S.Though it's far more volatile than markets in the U.S. and Europe, the Chinese stock market has also displayed impressive resilience. After the Shanghai composite index plunged more than $9 \%$ on Feb. 27, it quickly rebounded to new highs. Likewise, on Apr. 19, when the market benchmark saw a large slump on interest-rate worries, it recovered most of the losses by the next day. ${ }^{11}$

Ushad Subadar Agathee (2008) found the average returns of Stock Exchange of Mauritius (SEM) to be the lowest in the Month of March and Highest in the Month of June. The equality of means-return tests shows that returns were statistically the same across all months. The regression analysis reveals that returns were not independent of the Months of the Year, except for January. ${ }^{12}$

Selvarani.M and Leena Jenefa (2009) analyzed the trends in annual returns and daily returns. A set of parametric and non-parametric tests were employed to test the equality of mean returns and standard deviations of the returns. It was found that in the NSE, there was strong evidence of April and January Effect. After the introduction of the Rolling Settlement, Friday had become significant. As far as the Day Effect was concerned, Tuesday Effect was more prevalent than Monday Effect. ${ }^{13}$

Nageswari.P and Babu .M (2011) examined the Week End Effect in the Indian Stock Market. The study found that the mean returns were positive for all days of the week, highest on Friday and lowest on Monday. It was inferred that the Day of the Week Pattern did not exist in the Indian Stock Market during the study period. ${ }^{14}$

Nageswari .P and Selvam .M (2011) examined the Day of the Week Effect during the Post Rolling Settlement Period. The study found that the Highest Mean Return on Friday and the Lowest Mean Return on Tuesday were observed during the study period. Further, there was strong significant positive relationship between Monday - Friday and no significant relationship among other days of the week. The results indicated that the Day of the Week Effect did not exist in the Indian Stock Market during the study period the above literature provides an overview of Valuation of Seasonality Effects in various Stock Markets. An attempt has been made in this study to analyze the Stock Market Seasonality in India by taking the model from the above study. ${ }^{15}$

\section{Hypotheses Formulated}

In order to find out the dependence between successive market returns and stock returns of the selected companies, the following hypotheses have been framed. There is no inter-month effect on stock return in case of sample companies, there is no disparity in the mean returns across the days of the week, the means of the stock returns and market returns for five days are equal, daily series contains a unit root, monthly series contains a unit derivation and progress of stock returns and market returns is random.

\section{Result and discussion}

In order to find out the seasonal variation in the Indian stock market, the Dickey Fuller test and Phillip- Perron tests have been employed. The stock market returns are calculated as the natural log of daily and monthly relative mean value used for this study.

Following is the formula: 


$$
R t=\ln \left(\frac{P t}{P t}-1\right)
$$

Where: $\mathrm{Rt}=$ return on day' $\mathrm{t}^{\prime}$

$1 \mathrm{t}=$ index mean value on day' $\mathrm{t}$ '

lt- $1=$ index mean value on day' $t-1{ }^{\prime}$ and $l n=$ natural $\log$.

The results are given in Table 1. The positive returns in no of times have been found to be higher in BSE index and Grasim Industries Ltd about 1361 times and 1262 times respectively. The positive returns are also found to be the largest in case all companies except Bata India Ltd, Hindustan Petroleum Corporation Ltd, Satyam Computer Services Ltd and Wipro Ltd whose negative returns are higher than positive returns.

The day effect for the BSE index and companies is found by classifying mean return data into day wise. BSE index shows highest and lowest average returns on Thursdays and Tuesdays respectively. There is no strong evidence for week end effect in BSE index. While comparing the Monday mean return with Tuesday, Monday has higher mean return than Tuesday. This shows the presence of Tuesday effect for the entire index. For the selected pharmaceutical companies, 5 companies have negative mean return on Monday and positive mean return on Friday, it supports the Monday effect. Of 20 companies, 9 companies support the Tuesday effect. These companies have negative mean return on Tuesday and positive mean return on Monday. Most of the Tuesday mean return is lower than the Monday. Thursday effect is present in 12 companies. The results are given in Table 1.

BSE Daily

\begin{tabular}{|c|c|c|c|c|c|c|c|}
\hline Companies & $\mathrm{n}$ & Monday & Tuesday & Wednesday & Thursday & Friday & TOTAL \\
\hline \multirow[t]{2}{*}{ SENSEX } & no of positive returns & 273 & 277 & 270 & 277 & 264 & 1361 \\
\hline & no of negative returns & 225 & 221 & 230 & 221 & 226 & 1123 \\
\hline \multirow[t]{2}{*}{ ACC Ltd } & no of positive returns & 269 & 241 & 260 & 245 & 232 & 1247 \\
\hline & no of negative returns & 229 & 257 & 240 & 253 & 258 & 1237 \\
\hline \multirow[t]{2}{*}{ Ashok Leyland Ltd } & no of positive returns & 271 & 235 & 240 & 258 & 239 & 1243 \\
\hline & no of negative returns & 227 & 263 & 260 & 240 & 251 & 1241 \\
\hline \multirow[t]{2}{*}{ Bata India Ltd } & no of positive returns & 256 & 223 & 240 & 218 & 236 & 1173 \\
\hline & no of negative returns & 242 & 275 & 260 & 280 & 254 & 1311 \\
\hline \multirow[t]{2}{*}{ BHEL } & no of positive returns & 277 & 248 & 264 & 258 & 247 & 1294 \\
\hline & no of negative returns & 221 & 250 & 236 & 240 & 243 & 1190 \\
\hline \multirow[t]{2}{*}{ CIPLA Ltd } & no of positive returns & 258 & 253 & 238 & 261 & 260 & 1270 \\
\hline & no of negative returns & 240 & 245 & 262 & 237 & 230 & 1214 \\
\hline \multirow[t]{2}{*}{ Grasim Industries Ltd } & no of positive returns & 246 & 257 & 250 & 253 & 256 & 1262 \\
\hline & no of negative returns & 252 & 241 & 250 & 245 & 234 & 1222 \\
\hline \multirow[t]{2}{*}{ HDFC Bank Ltd } & no of positive returns & 257 & 237 & 256 & 265 & 247 & 1262 \\
\hline & no of negative returns & 241 & 261 & 244 & 233 & 243 & 1222 \\
\hline \multirow{2}{*}{$\begin{array}{l}\text { Hero Honda Motors } \\
\text { Ltd }\end{array}$} & no of positive returns & 259 & 244 & 246 & 246 & 250 & 1245 \\
\hline & no of negative returns & 239 & 254 & 254 & 252 & 240 & 1239 \\
\hline \multirow{2}{*}{$\begin{array}{l}\text { Hindustan Petroleum } \\
\text { Corporation Ltd }\end{array}$} & no of positive returns & 253 & 266 & 244 & 234 & 230 & 1227 \\
\hline & no of negative returns & 245 & 232 & 256 & 264 & 260 & 1257 \\
\hline \multirow[t]{2}{*}{ ICICI Bank Ltd } & no of positive returns & 256 & 253 & 247 & 246 & 253 & 1255 \\
\hline & no of negative returns & 242 & 245 & 253 & 252 & 237 & 1229 \\
\hline \multirow{2}{*}{$\begin{array}{l}\text { Infosys Technologies } \\
\text { Ltd }\end{array}$} & no of positive returns & 263 & 258 & 244 & 242 & 245 & 1252 \\
\hline & no of negative returns & 235 & 240 & 256 & 256 & 245 & 1232 \\
\hline \multirow{2}{*}{ ITC LTD } & no of positive returns & 269 & 273 & 261 & 269 & 259 & 1331 \\
\hline & no of negative returns & 229 & 225 & 239 & 229 & 231 & 1153 \\
\hline \multirow[t]{2}{*}{ M\&M LTD } & no of positive returns & 264 & 244 & 256 & 247 & 251 & 1262 \\
\hline & no of negative returns & 234 & 254 & 244 & 251 & 239 & 1222 \\
\hline \multirow[t]{2}{*}{ ONCG LTD } & no of positive returns & 283 & 230 & 280 & 250 & 246 & 1289 \\
\hline & no of negative returns & 215 & 268 & 220 & 248 & 244 & 1195 \\
\hline \multirow{2}{*}{$\begin{array}{l}\text { Ranbaxy Laboratories } \\
\text { Ltd }\end{array}$} & no of positive returns & 246 & 252 & 256 & 253 & 246 & 1253 \\
\hline & no of negative returns & 252 & 246 & 244 & 245 & 244 & 1231 \\
\hline \multirow{2}{*}{$\begin{array}{l}\text { Reliance Industries } \\
\text { Ltd }\end{array}$} & no of positive returns & 248 & 259 & 246 & 260 & 237 & 1250 \\
\hline & no of negative returns & 250 & 239 & 254 & 238 & 253 & 1234 \\
\hline \multirow{2}{*}{$\begin{array}{l}\text { Sat yam Computer } \\
\text { Services Ltd }\end{array}$} & no of positive returns & 238 & 243 & 259 & 245 & 245 & 1230 \\
\hline & no of negative returns & 260 & 255 & 241 & 253 & 245 & 1254 \\
\hline \multirow[t]{2}{*}{ Wipro Ltd } & no of positive returns & 235 & 252 & 259 & 250 & 237 & 1233 \\
\hline & no of negative returns & 263 & 246 & 241 & 248 & 253 & 1251 \\
\hline \multicolumn{8}{|l|}{ no of positive returns } \\
\hline \multicolumn{8}{|l|}{ no of negative returns } \\
\hline Highest positive & & 10 & 4 & 5 & 4 & 0 & 20 \\
\hline
\end{tabular}


Stock Market Seasonality- Time Varying Volatility In The Emerging Indian Stock Market

\begin{tabular}{|l|l|l|l|l|l|l|l|}
\hline returns & & & & & & & \\
\hline $\begin{array}{l}\text { Highest positive } \\
\text { returns }\end{array}$ & & & & & & & \\
\hline
\end{tabular}

Source: Computed data

Table - 2

NSE Daily

\begin{tabular}{|c|c|c|c|c|c|c|c|}
\hline Companies & $\mathrm{n}$ & Monday & Tuesday & Wednesday & Thursday & Friday & TOTAL \\
\hline \multirow{2}{*}{ NIFTY } & no of positive returns & 274 & 270 & 283 & 272 & 268 & 1367 \\
\hline & no of negative returns & 224 & 228 & 217 & 226 & 222 & 1117 \\
\hline \multirow{2}{*}{ ACC Ltd } & no of positive returns & 270 & 246 & 259 & 248 & 248 & 1271 \\
\hline & no of negative returns & 228 & 252 & 241 & 250 & 242 & 1213 \\
\hline \multirow{2}{*}{ Ashok Leyland Ltd } & no of positive returns & 263 & 232 & 261 & 237 & 240 & 1233 \\
\hline & no of negative returns & 235 & 266 & 239 & 261 & 250 & 1251 \\
\hline \multirow{2}{*}{ Bata India Ltd } & no of positive returns & 253 & 207 & 252 & 209 & 226 & 1147 \\
\hline & no of negative returns & 245 & 291 & 248 & 289 & 264 & 1337 \\
\hline \multirow{2}{*}{ BHEL } & no of positive returns & 261 & 255 & 268 & 244 & 244 & 1272 \\
\hline & no of negative returns & 237 & 243 & 232 & 254 & 246 & 1212 \\
\hline \multirow{2}{*}{ CIPLA Ltd } & no of positive returns & 256 & 254 & 255 & 249 & 249 & 1263 \\
\hline & no of negative returns & 242 & 244 & 245 & 249 & 241 & 1221 \\
\hline \multirow{2}{*}{ Grasim Industries Ltd } & no of positive returns & 245 & 254 & 264 & 243 & 249 & 1255 \\
\hline & no of negative returns & 253 & 244 & 236 & 255 & 241 & 1229 \\
\hline \multirow{2}{*}{ HDFC Bank Ltd } & no of positive returns & 251 & 264 & 251 & 235 & 251 & 1252 \\
\hline & no of negative returns & 247 & 234 & 249 & 263 & 239 & 1232 \\
\hline \multirow{2}{*}{$\begin{array}{l}\text { Hero Honda Motors } \\
\text { Ltd }\end{array}$} & no of positive returns & 254 & 252 & 267 & 251 & 254 & 1278 \\
\hline & no of negative returns & 244 & 246 & 233 & 247 & 236 & 1206 \\
\hline \multirow{2}{*}{$\begin{array}{l}\text { Hindustan Petroleum } \\
\text { Corporation Ltd }\end{array}$} & no of positive returns & 244 & 264 & 243 & 241 & 231 & 1223 \\
\hline & no of negative returns & 254 & 234 & 257 & 257 & 259 & 1261 \\
\hline \multirow{2}{*}{ ICICI Bank Ltd } & no of positive returns & 253 & 250 & 261 & 246 & 253 & 1263 \\
\hline & no of negative returns & 245 & 248 & 239 & 252 & 237 & 1221 \\
\hline \multirow{2}{*}{$\begin{array}{l}\text { Infosys Technologies } \\
\text { Ltd }\end{array}$} & no of positive returns & 243 & 245 & 256 & 255 & 253 & 1252 \\
\hline & no of negative returns & 255 & 253 & 244 & 243 & 237 & 1232 \\
\hline \multirow{2}{*}{ ITC LTD } & no of positive returns & 240 & 246 & 271 & 246 & 245 & 1248 \\
\hline & no of negative returns & 258 & 252 & 229 & 252 & 245 & 1236 \\
\hline \multirow{2}{*}{ M\&M LTD } & no of positive returns & 268 & 247 & 258 & 246 & 256 & 1275 \\
\hline & no of negative returns & 230 & 251 & 242 & 252 & 234 & 1209 \\
\hline \multirow{2}{*}{ ONCG LTD } & no of positive returns & 275 & 225 & 290 & 241 & 236 & 1267 \\
\hline & no of negative returns & 223 & 273 & 210 & 257 & 254 & 1217 \\
\hline \multirow{2}{*}{$\begin{array}{l}\text { Ranbaxy Laboratories } \\
\text { Ltd }\end{array}$} & no of positive returns & 260 & 234 & 265 & 244 & 250 & 1253 \\
\hline & no of negative returns & 238 & 264 & 235 & 254 & 240 & 1231 \\
\hline \multirow{2}{*}{$\begin{array}{l}\text { Reliance Industries } \\
\text { Ltd }\end{array}$} & no of positive returns & 261 & 270 & 265 & 267 & 257 & 1320 \\
\hline & no of negative returns & 237 & 228 & 235 & 231 & 233 & 1164 \\
\hline \multirow{2}{*}{$\begin{array}{l}\text { Sat yam Computer } \\
\text { Services Ltd }\end{array}$} & no of positive returns & 241 & 250 & 243 & 258 & 235 & 1227 \\
\hline & no of negative returns & 257 & 248 & 257 & 240 & 255 & 1257 \\
\hline \multirow{2}{*}{ Wipro Ltd } & no of positive returns & 232 & 252 & 270 & 251 & 228 & 1233 \\
\hline & no of positive returns & 266 & 246 & 230 & 247 & 262 & 1251 \\
\hline
\end{tabular}

Source: Computed data 
Stock Market Seasonality- Time Varying Volatility In The Emerging Indian Stock Market

Table - 3 BSE data for Month wise for 10 years

\begin{tabular}{|c|c|c|c|c|c|c|c|c|c|c|c|c|c|}
\hline \multirow{3}{*}{ SENSEX } & $\mathrm{n}$ & April & May & June & July & August & September & October & November & December & January & February & March \\
\hline & no of positive returns & 105 & 124 & 119 & 115 & 123 & 117 & 106 & 119 & 123 & 109 & 104 & 99 \\
\hline & no of negative returms & 91 & 90 & 97 & 104 & 91 & 91 & 103 & 85 & 86 & 102 & 93 & 107 \\
\hline \multirow{2}{*}{ ACCLtd } & no of positive returns & 105 & 101 & 101 & 115 & 122 & 109 & 105 & 122 & 113 & 103 & 101 & 97 \\
\hline & no of negative retums & 91 & 113 & 115 & 104 & 92 & 99 & 104 & 82 & 96 & 108 & 96 & 109 \\
\hline \multirow{2}{*}{ Ashok Leyland Ltd } & no of positive returns & 100 & 106 & 101 & 111 & 110 & 101 & 105 & 115 & 110 & 96 & 105 & 93 \\
\hline & no of negative retums & 96 & 108 & 115 & 108 & 104 & 107 & 104 & 89 & 99 & 115 & 92 & 113 \\
\hline \multirow{2}{*}{ Bata India Ltd } & no of positive returns & 96 & 101 & 100 & 108 & 110 & 92 & 104 & 101 & 95 & 86 & 86 & 96 \\
\hline & no of negative returms & 100 & 113 & 116 & 111 & 104 & 116 & 105 & 103 & 114 & 125 & 111 & 110 \\
\hline \multirow{2}{*}{ BHEL } & no of positive returns & 101 & 115 & 109 & 112 & 114 & 111 & 111 & 106 & 110 & 112 & 94 & 99 \\
\hline & no of negative returns & 95 & 99 & 107 & 107 & 100 & 97 & 98 & 98 & 99 & 99 & 103 & 107 \\
\hline \multirow{2}{*}{ CIPLALtd } & no of positive returns & 102 & 106 & 105 & 122 & 116 & 109 & 111 & 112 & 101 & 92 & 96 & 107 \\
\hline & no of negative returns & 94 & 108 & 111 & 97 & 98 & 99 & 98 & 92 & 108 & 119 & 101 & 99 \\
\hline \multirow{2}{*}{ Grasim Industries Ltd } & no of positive returns & 106 & 96 & 106 & 123 & 115 & 109 & 105 & 112 & 112 & 99 & 94 & 96 \\
\hline & no of negative retums & 90 & 118 & 110 & 96 & 99 & 99 & 104 & 92 & 97 & 112 & 103 & 110 \\
\hline \multirow{2}{*}{ HDFC BankLtd } & no of positive returns & 99 & 103 & 114 & 112 & 109 & 110 & 111 & 111 & 109 & 101 & 93 & 94 \\
\hline & no of negative returns & 97 & 111 & 102 & 107 & 105 & 98 & 98 & 93 & 100 & 110 & 104 & 112 \\
\hline \multirow{2}{*}{ Hero Honda Motors Ltd } & no of positive returns & 92 & 110 & 118 & 118 & 103 & 101 & 104 & 111 & 108 & 109 & 87 & 93 \\
\hline & no of negative returns & 104 & 104 & 98 & 101 & 111 & 107 & 105 & 93 & 101 & 102 & 110 & 113 \\
\hline \multirow{2}{*}{ Hindustan Petroleum Corporation Ltd } & no of positive returns & 90 & 103 & 102 & 111 & 111 & 107 & 102 & 111 & 115 & 99 & 97 & 89 \\
\hline & no of negative retums & 106 & 111 & 114 & 108 & 103 & 101 & 107 & 93 & 94 & 112 & 100 & 117 \\
\hline \multirow{2}{*}{ ICICIBank Ltd } & no of positive retums & 98 & 99 & 100 & 120 & 118 & 113 & 94 & 100 & 125 & 108 & 95 & 90 \\
\hline & no of negative returns & 98 & 115 & 116 & 99 & 96 & 95 & 115 & 104 & 84 & 103 & 102 & 116 \\
\hline \multirow{2}{*}{ Infosys Technologies Ltd } & no of positive returns & 97 & 117 & 101 & 107 & 100 & 105 & 111 & 105 & 116 & 105 & 102 & 95 \\
\hline & no of negative returms & 99 & 97 & 115 & 112 & 114 & 103 & 98 & 99 & 93 & 106 & 95 & 111 \\
\hline \multirow{2}{*}{ ITCLTD } & no of positive retums & 107 & 119 & 109 & 116 & 121 & 116 & 105 & 105 & 113 & 105 & 107 & 113 \\
\hline & no of negative returms & 89 & 95 & 107 & 103 & 93 & 92 & 104 & 99 & 96 & 106 & 90 & 93 \\
\hline \multirow{2}{*}{ M\&MLTD } & no of positive returns & 92 & 104 & 110 & 110 & 107 & 98 & 116 & 116 & 123 & 101 & 99 & 95 \\
\hline & no of negative returns & 104 & 110 & 106 & 109 & 107 & 110 & 93 & 88 & 86 & 110 & 98 & 111 \\
\hline \multirow{2}{*}{ ONCGLTD } & no of positive returms & 94 & 119 & 109 & 115 & 108 & 114 & 104 & 109 & 107 & 116 & 95 & 110 \\
\hline & no of negative returms & 102 & 95 & 107 & 104 & 106 & 94 & 105 & 100 & 102 & 95 & 102 & 96 \\
\hline \multirow{2}{*}{ Ranbaxy Laboratories Ltd } & no of positive returns & 89 & 110 & 111 & 116 & 120 & 103 & 106 & 113 & 113 & 99 & 92 & 87 \\
\hline & no of negative returns & 107 & 104 & 105 & 103 & 94 & 105 & 103 & 91 & 96 & 112 & 105 & 119 \\
\hline \multirow{2}{*}{ Reliance Industries Ltd } & no of positive returms & 91 & 115 & 107 & 114 & 114 & 98 & 107 & 112 & 108 & 102 & 92 & 96 \\
\hline & no of negative returms & 105 & 99 & 109 & 105 & 100 & 110 & 102 & 97 & 101 & 109 & 105 & 110 \\
\hline \multirow{2}{*}{ Satyam Computer Services Ltd } & no of positive returns & 99 & 104 & 110 & 97 & 116 & 88 & 101 & 106 & 114 & 98 & 103 & 102 \\
\hline & no of negative retums & 97 & 110 & 106 & 122 & 98 & 120 & 108 & 98 & 95 & 113 & 94 & 104 \\
\hline \multirow{2}{*}{ Wipro Ltd } & no of positive returns & 85 & 111 & 117 & 95 & 126 & 99 & 97 & 116 & 111 & 108 & 92 & 86 \\
\hline & no of positive returms & 111 & 103 & 99 & 124 & 88 & 109 & 112 & 88 & 98 & 103 & 105 & 120 \\
\hline
\end{tabular}

c Source: Computed data

Table - 4 NSE data for Month wise for 10 years

\begin{tabular}{|c|c|c|c|c|c|c|c|c|c|c|c|c|c|}
\hline Companies NSE & $\mathrm{n}$ & April & May & June & July & August & September & October & November & \begin{tabular}{|l|} 
December \\
\end{tabular} & January & February & March \\
\hline \multirow{2}{*}{ SENSEX } & no of positive retums & 109 & 118 & \begin{tabular}{l|l}
118 \\
\end{tabular} & 118 & 126 & 114 & 105 & 128 & 125 & 103 & 103 & 104 \\
\hline & no of negative returns & 87 & 96 & 98 & 101 & 88 & 94 & 104 & 76 & 84 & 108 & 94 & 102 \\
\hline \multirow{2}{*}{ ACCLtd } & no of positive returms & 102 & 97 & 105 & 118 & 122 & 110 & 98 & 122 & 113 & 101 & 91 & 95 \\
\hline & no of negative returns & 94 & 117 & 111 & 101 & 92 & 98 & 111 & 82 & 96 & 110 & 106 & 111 \\
\hline \multirow{2}{*}{ Ashok Leyland Ltd } & no of positive returns & 103 & 108 & 112 & 113 & 110 & 98 & 101 & 109 & 113 & 88 & 109 & 90 \\
\hline & no of negative returns & 93 & 106 & 114 & 106 & 104 & 110 & 108 & 95 & 96 & 123 & 88 & 116 \\
\hline \multirow{2}{*}{ Bata India Ltd } & no of positive retums & 99 & 102 & 98 & 93 & 111 & 89 & 103 & 100 & 102 & 79 & 81 & 96 \\
\hline & no of negative returns & 97 & 112 & \begin{tabular}{l|l|}
118 \\
\end{tabular} & 126 & 103 & 119 & 106 & 104 & 107 & 132 & 116 & 110 \\
\hline \multirow{2}{*}{ BHEL } & no of positive returns & 98 & 112 & 110 & 113 & 117 & 103 & 102 & 112 & 106 & 107 & 98 & 94 \\
\hline & no of negative returns & 98 & 102 & 106 & 106 & 97 & 105 & 107 & 97 & 103 & 104 & 99 & 112 \\
\hline \multirow{2}{*}{ CIPLALtd } & no of positive returns & 99 & 105 & 103 & 116 & 125 & 107 & 107 & 115 & 100 & 91 & 94 & 109 \\
\hline & no of negative returns & 97 & 109 & 113 & 103 & 89 & 101 & 112 & 89 & 109 & 120 & 103 & 97 \\
\hline \multirow{2}{*}{ Grasim Industries Ltd } & no of positive returns & 103 & 97 & 105 & 119 & 124 & 105 & 104 & 107 & 113 & 103 & 93 & 92 \\
\hline & no of negative returns & 93 & 117 & 111 & 100 & 90 & 103 & 105 & 97 & 96 & 108 & 104 & 114 \\
\hline \multirow{2}{*}{ HDFC Bank Ltd } & no of positive returns & 105 & 101 & 108 & 121 & 106 & 113 & 97 & 100 & 105 & 114 & 98 & 92 \\
\hline & no of negative returns & 91 & 113 & \begin{tabular}{l|l|}
108 \\
\end{tabular} & 98 & 108 & 95 & 112 & 104 & 104 & 97 & 99 & 114 \\
\hline \multirow{2}{*}{ Hero HondaMotors Ltd } & no of positive returns & 101 & 108 & 117 & 118 & 109 & 107 & 105 & 114 & 111 & 106 & 86 & 100 \\
\hline & no of negative returns & 95 & 106 & 99 & \begin{tabular}{l|l}
101 \\
\end{tabular} & 105 & 101 & 104 & 90 & 98 & 105 & 111 & 106 \\
\hline \multirow{2}{*}{ Hindustan Petroleum Corporation Ltd } & no of positive returms & 88 & 103 & 105 & 109 & 106 & 102 & 100 & 114 & 115 & 95 & 104 & 93 \\
\hline & no of negative returns & 108 & 111 & 111 & 110 & 108 & 106 & 109 & 90 & 94 & 116 & 93 & 113 \\
\hline \multirow{2}{*}{ ICICIBank Ltd } & no of positive returns & 99 & 114 & 99 & 108 & 100 & 107 & 110 & 109 & 113 & 108 & 101 & 101 \\
\hline & no of negative returns & 97 & 100 & 117 & 111 & 114 & 101 & 99 & 95 & 96 & 103 & 96 & 105 \\
\hline \multirow{2}{*}{ Infosys Technologies Ltd } & no of positive returns & 90 & 110 & 115 & 115 & 117 & 100 & 105 & 110 & 112 & 98 & 92 & 92 \\
\hline & no of negative returms & 106 & 104 & 101 & 104 & 97 & 108 & 104 & 94 & 97 & 113 & 105 & 114 \\
\hline \multirow{2}{*}{ ITCLTD } & no of positive returns & 102 & 104 & 108 & 98 & 116 & 91 & 100 & 105 & 114 & 104 & 107 & 105 \\
\hline & no of negative returns & 94 & 110 & 108 & 121 & 98 & 117 & 109 & 99 & 95 & 107 & 90 & 101 \\
\hline \multirow{2}{*}{ M\&MLTD } & no of positive returns & 93 & 104 & 112 & 118 & 106 & 98 & 108 & 122 & 119 & 106 & 99 & 99 \\
\hline & no of negative returns & 103 & 110 & 104 & 101 & 108 & 110 & 101 & 82 & 90 & 105 & 98 & 107 \\
\hline \multirow{2}{*}{ ONCGLTD } & no of positive returms & 95 & 114 & 112 & \begin{tabular}{ll|}
117 \\
\end{tabular} & 107 & 111 & 101 & 108 & 102 & 110 & 92 & 110 \\
\hline & no of negative returns & 101 & 100 & 104 & 102 & 107 & 97 & 108 & 96 & 107 & 101 & 105 & 96 \\
\hline \multirow{2}{*}{ Ranbaxy Laboratories Ltd } & no of positive returns & 99 & 99 & 102 & 124 & 121 & 99 & 93 & 104 & 121 & 104 & 94 & 96 \\
\hline & no of negative returns & 97 & 115 & 114 & 95 & 93 & 109 & 116 & 100 & 88 & 107 & 103 & 110 \\
\hline \multirow{2}{*}{ Reliance Industries Ltd } & no of positive eeturns & 107 & 113 & 106 & 118 & 116 & 116 & 102 & 110 & 113 & 104 & 111 & 109 \\
\hline & no of negative returns & 89 & 101 & 110 & 101 & 98 & 92 & 107 & 94 & 96 & 107 & 86 & 97 \\
\hline \multirow{2}{*}{ Satyam Computer Services Ltd } & no of positive eeturms & 88 & 107 & 106 & 112 & 114 & 95 & 102 & 110 & 110 & 100 & 95 & 103 \\
\hline & no of negative returns & 108 & 107 & 110 & 107 & 100 & 113 & 107 & 94 & 99 & 111 & 102 & 113 \\
\hline \multirow{2}{*}{ WiproLtd } & no of positive returns & 81 & 105 & 118 & 95 & 123 & 96 & 100 & 116 & 114 & 106 & 92 & 93 \\
\hline & no of positive returns & 115 & 109 & 98 & 124 & 91 & 112 & 109 & 88 & 95 & 105 & 105 & 113 \\
\hline
\end{tabular}

Source: Computed data 
Monthly returns of the sample companies listed at both BSE and NSE. The monthly returns are calculated as positive returns and negative returns. These monthly returns are calculated are given in the above two table's one for BSE and another for NSE.

As far as BSE is concerned, the highest positive returns is recorded for (1336) ITC limited and this is followed by ONCG limited which has a positive return (1300). The highest negative return is calculated for Bata India limited and this is followed by Hindustan Petroleum Corporation limited.

Regarding NSE, the highest positive returns (1325) goes to Reliance Industries limited and M\&M limited placed at the second highest positive scorer, besides the highest negative return goes to Bata India limited (1350) and Reliance industries scored (1178) and occupied the second highest negative scorer.

\section{Result of chi-square}

\section{Testing Of Hypotheses}

Further chi-square was applied to examine the hypothesis that there is any seasonal or inter month effect or not .For this purpose, first a contingency table consisting of months showing positive returns \& negative returns between two sets of months were prepared. The first set includes May to September and November to February. In contrast the second set comprises the month showing poor growth or negative returns as concluded in Table 4.4.1.These months include April, October and March.

$\mathrm{H}_{\mathrm{O}}$ : There is no inter -month or inter-day effect on Stock Return in case of sample companies.

$\mathrm{H}_{1}$ : There is an inter month effect on Stock Return in case of sample companies.

Calculated chi square value $=(\mathrm{O}-\mathrm{E})^{2} / \mathrm{E}=12.73$

With degrees of freedom $(\mathrm{c}-1) \times(\mathrm{r}-1)$ i.e $1 \times 1=1$, critical value is 3.841 . Since the calculated value is greater than the critical value; Null Hypothesis is rejected at $5 \%$ level of significance. It implies that there is an inter month effect on stock return in case of sample companies.

Table - 5
Chi - Square
NSE DAILY
\begin{tabular}{|c|l|c|c|c|c|c|}
\hline $\begin{array}{l}\text { C. } \\
\text { No }\end{array}$ & \multicolumn{1}{|c|}{ Company Name } & $\begin{array}{c}\text { Chi - square } \\
\text { Calculated }\end{array}$ & D.F & $\begin{array}{c}\text { Chi - square } \\
\text { Table value }\end{array}$ & P - Value & Inferences \\
\hline 2 & ACC Ltd & 1.64 & 1 & 3.84 & 0.20 & $\mathrm{H}_{\mathrm{O}}:$ Accept \\
\hline 3 & Ashok Leyland Ltd & 5.49 & 1 & 3.84 & 0.02 & $\mathrm{H}_{\mathrm{O}}:$ Reject \\
\hline 4 & Bata India Ltd & 13.15 & 1 & 3.84 & 0.00 & $\mathrm{H}_{\mathrm{O}}:$ Reject \\
\hline 5 & BHEL & 2.16 & 1 & 3.84 & 0.14 & $\mathrm{H}_{\mathrm{O}}:$ Accept \\
\hline 6 & CIPLA Ltd & 0.18 & 1 & 3.84 & 0.67 & $\mathrm{H}_{\mathrm{O}}:$ Accept \\
\hline 7 & Grasim Industries Ltd & 1.55 & 1 & 3.84 & 0.21 & $\mathrm{H}_{\mathrm{O}}:$ Accept \\
\hline 8 & HDFC Bank Ltd & 2.57 & 1 & 3.84 & 0.11 & $\mathrm{H}_{\mathrm{O}}:$ Accept \\
\hline 9 & Hero Honda Motors Ltd & 2.87 & 1 & 3.84 & 0.27 & $\mathrm{H}_{\mathrm{O}}:$ Accept \\
\hline 10 & Hindustan Petroleum Corporation & 3.55 & 1 & 3.84 & 0.06 & $\mathrm{H}_{\mathrm{O}}:$ Accept \\
\hline & Ltd & & & & \\
\hline 11 & ICICI Bank Ltd & 1.32 & 1 & 3.84 & 0.25 & $\mathrm{H}_{\mathrm{O}}:$ Accept \\
\hline 12 & Infosys Technologies Ltd & 2.33 & 1 & 3.84 & 0.13 & $\mathrm{H}_{\mathrm{O}}:$ Accept \\
\hline 13 & ITC LTD & 2.23 & 1 & 3.84 & 0.14 & $\mathrm{H}_{\mathrm{O}}:$ Accept \\
\hline 14 & M\&M LTD & 20.99 & 1 & 3.84 & 0.00 & $\mathrm{H}_{\mathrm{O}} ;$ Reject \\
\hline 15 & ONCG LTD & 3.99 & 1 & 3.84 & 0.05 & $\mathrm{H}_{\mathrm{O}}:$ Reject \\
\hline 16 & Ranbaxy Laboratories Ltd & 3.01 & 1 & 3.84 & 0.31 & $\mathrm{H}_{\mathrm{O}}:$ Accept \\
\hline 17 & Reliance Industries Ltd & 1.72 & 1 & 3.84 & 0.19 & $\mathrm{H}_{\mathrm{O}}:$ Accept \\
\hline 18 & Sat yam Computer Services Ltd & 6.22 & 1 & 3.84 & 0.01 & $\mathrm{H}_{\mathrm{O}}:$ Reject \\
\hline 19 & Wipro Ltd & & & & & \\
\hline
\end{tabular}

Source: Computed data

Calculated value of chi-square and table value of chi-square and other descriptive statistics regarding the chi-square. These results have been derived to test the hypotheses for NSE daily analysis. The null hypothesis is framed as there is no inter-day effect on stock returns of the sample companies. As per this analysis, there is no inter-day effect on stock returns of ACC limited, BHEL, CIPLA limited, Grasim Industries, HDFC Bank Limited, Hero Honda motors Limited, Hindustan petroleum limited, ICICI bank limited, Infosys technologies, ITC Limited, M\&M limited, Reliance Industries and Sathyam computer services. For other companies, there is existence of inter-day effect on stock returns; these have been done at 5 per cent level of significance. 
Stock Market Seasonality- Time Varying Volatility In The Emerging Indian Stock Market

\begin{tabular}{|c|c|c|c|c|c|c|}
\hline \multicolumn{7}{|c|}{ Table -6 BSE DAILY } \\
\hline S. No & Company Name & Chi-square & D.F & $\begin{array}{l}\text { Chi - square } \\
\text { Table value }\end{array}$ & $\begin{array}{c}\mathbf{P}- \\
\text { Value }\end{array}$ & Inferences \\
\hline 2 & ACC Ltd & 5.25 & 1 & 3.84 & 0.02 & $\mathrm{H}_{\mathrm{O}}:$ Reject \\
\hline 3 & Ashok Leyland Ltd & 6.28 & 1 & 3.84 & 0.01 & $\mathrm{H}_{\mathrm{O}}$ :Reject \\
\hline 4 & Bata India Ltd & 4.37 & 1 & 3.84 & 0.04 & $\mathrm{H}_{\mathrm{O}}$ :Reject \\
\hline 5 & BHEL & 1.31 & 1 & 3.84 & 0.25 & $\mathrm{H}_{\mathrm{O}}$ :Accept \\
\hline 6 & CIPLA Ltd & 3.12 & 1 & 3.84 & 0.08 & $\mathrm{H}_{\mathrm{O}}:$ Accept \\
\hline 7 & Grasim Industries Ltd & 0.49 & 1 & 3.84 & 0.48 & $\mathrm{H}_{\mathrm{O}}$ :Accept \\
\hline 8 & HDFC Bank Ltd & 2.58 & 1 & 3.84 & 0.11 & $\mathrm{H}_{\mathrm{O}}$ :Accept \\
\hline 9 & Hero Honda Motors Ltd & 1.28 & 1 & 3.84 & 0.26 & $\mathrm{H}_{\mathrm{O}}$ :Accept \\
\hline 10 & $\begin{array}{l}\text { Hindustan Petroleum } \\
\text { Corporation Ltd }\end{array}$ & 4.89 & 1 & 3.84 & 0.03 & $\mathrm{H}_{\mathrm{O}}:$ Reject \\
\hline 11 & ICICI Bank Ltd & 0.84 & 1 & 3.84 & 0.36 & $\mathrm{H}_{\mathrm{O}}$ :Accept \\
\hline 12 & Infosys Technologies Ltd & 1.94 & 1 & 3.84 & 0.16 & $\mathrm{H}_{\mathrm{O}}$ :Accept \\
\hline 13 & ITC LTD & 2.87 & 1 & 3.84 & 0.32 & $\mathrm{H}_{\mathrm{O}}:$ Accept \\
\hline 14 & M\&M LTD & 1.51 & 1 & 3.84 & 0.22 & $\mathrm{H}_{\mathrm{O}}$ :Accept \\
\hline 15 & ONCG LTD & 8.13 & 1 & 3.84 & 0.00 & $\mathrm{H}_{\mathrm{O}}:$ Reject \\
\hline 16 & Ranbaxy Laboratories Ltd & 0.27 & 1 & 3.84 & 0.60 & $\mathrm{H}_{\mathrm{O}}:$ Accept \\
\hline 17 & Reliance Industries Ltd & 1.12 & 1 & 3.84 & 0.15 & $\mathrm{H}_{\mathrm{O}}$ :Accept \\
\hline 18 & $\begin{array}{l}\text { Sat yam Computer Services } \\
\text { Ltd }\end{array}$ & 1.28 & 1 & 3.84 & 0.26 & $\mathrm{H}_{\mathrm{O}}$ :Accept \\
\hline 19 & Wipro Ltd & 2.28 & 1 & 3.84 & 0.13 & $\mathrm{H}_{\mathrm{O}}$ :Accept \\
\hline
\end{tabular}

Source: Computed

Regarding the inter-day effect on stock returns in BSE, there is existence of inter-day effect on returns of ACC limited, Ashok Leyland Limited, Bata India limited, Hindustan Petroleum limited, ONCG limited, since the calculated values of chi-square are more than the table values of Chi-square at 5 per cent level of significance.

Table - 7 NSE Monthly

\begin{tabular}{|c|c|c|c|c|c|c|}
\hline S. No & Company Name & Chi - square & D.F & $\begin{array}{l}\text { Chi - square } \\
\text { Table value }\end{array}$ & P - Value & Inferences \\
\hline 1 & NIFTY & 3.30 & 1 & 3.84 & 0.07 & $\mathrm{H}_{\mathrm{O}}:$ Accept \\
\hline 2 & ACC Ltd & 16.48 & 1 & 3.84 & 0.00 & $\mathrm{H}_{\mathrm{O}}:$ Reject \\
\hline 3 & Ashok Leyland Ltd & 12.05 & 1 & 3.84 & 0.00 & $\mathrm{H}_{\mathrm{O}}$ :Reject \\
\hline 4 & Bata India Ltd & 5.24 & 1 & 3.84 & 0.02 & $\mathrm{H}_{\mathrm{O}}:$ Reject \\
\hline 5 & BHEL & 2.82 & 1 & 3.84 & 0.09 & $\mathrm{H}_{\mathrm{O}}:$ Accept \\
\hline 6 & CIPLA Ltd & 9.82 & 1 & 3.84 & 0.00 & $\mathrm{H}_{\mathrm{O}}$ :Reject \\
\hline 7 & Grasim Industries Ltd & 9.85 & 1 & 3.84 & 0.00 & $\mathrm{H}_{\mathrm{O}}:$ Reject \\
\hline 8 & HDFC Bank Ltd & 6.64 & 1 & 3.84 & 0.01 & $\mathrm{H}_{\mathrm{O}}$ :Reject \\
\hline 9 & Hero Honda Motors Ltd & 4.93 & 1 & 3.84 & 0.03 & $\mathrm{H}_{\mathrm{O}}$ :Reject \\
\hline 10 & $\begin{array}{l}\text { Hindustan Petroleum } \\
\text { Corporation Ltd }\end{array}$ & 9.03 & 1 & 3.84 & 0.00 & $\mathrm{H}_{\mathrm{O}}:$ Reject \\
\hline 11 & ICICI Bank Ltd & 4.61 & 1 & 3.84 & 0.03 & $\mathrm{H}_{\mathrm{O}}$ :Reject \\
\hline 12 & Infosys Technologies Ltd & 9.99 & 1 & 3.84 & 0.00 & $\mathrm{H}_{\mathrm{O}}:$ Reject \\
\hline 13 & ITC LTD & 7.96 & 1 & 3.84 & 0.01 & $\mathrm{H}_{\mathrm{O}}:$ Reject \\
\hline 14 & M\&M LTD & 6.95 & 1 & 3.84 & 0.01 & $\mathrm{H}_{\mathrm{O}}$ :Reject \\
\hline 15 & ONCG LTD & 4.74 & 1 & 3.84 & 0.03 & $\mathrm{H}_{\mathrm{O}}:$ Reject \\
\hline 16 & Ranbaxy Laboratories Ltd & 13.99 & 1 & 3.84 & 0.00 & $\mathrm{H}_{\mathrm{O}}:$ Reject \\
\hline 17 & Reliance Industries Ltd & 5.15 & 1 & 3.84 & 0.02 & $\mathrm{H}_{\mathrm{O}}:$ Reject \\
\hline 18 & $\begin{array}{l}\text { Sat yam Computer Services } \\
\text { Ltd }\end{array}$ & 6.40 & 1 & 3.84 & 0.01 & $\mathrm{H}_{\mathrm{O}}:$ Reject \\
\hline 19 & Wipro Ltd & 20.02 & 1 & 3.84 & 0.00 & $\mathrm{H}_{\mathrm{O}}:$ Reject \\
\hline
\end{tabular}

Source: Computed data

As far as inter- month effect on returns of the selected companies at NSE is concerned, the calculated
f chi-square are less than the table value of chi-square for BHEL only. Therefore, it is concluded that values of chi-square are less than the table value of chi-square for BHEL only. Therefore, it is concluded that
there is no existence of inter-month effect of returns of BHEL limited. For other companies, the calculated values of chi-square are more than the table values of chi-square. Hence, it is found that there is existence of inter-month effect on returns of the companies. 
Table - 8 BSE Monthly

\begin{tabular}{|c|c|c|c|c|c|c|}
\hline S. No & Company Name & Chi - square & D.F & $\begin{array}{l}\text { Chi - square } \\
\text { Table value }\end{array}$ & P - Value & Inferences \\
\hline 1 & NIFTY & 3.70 & 1 & 3.84 & 0.05 & $\mathrm{H}_{\mathrm{O}}$ :Accept \\
\hline 2 & ACC Ltd & 9.20 & 1 & 3.84 & 0.00 & $\mathrm{H}_{\mathrm{O}}$ :Reject \\
\hline 3 & Ashok Leyland Ltd & 6.35 & 1 & 3.84 & 0.01 & $\mathrm{H}_{\mathrm{O}}:$ Reject \\
\hline 4 & Bata India Ltd & 1.87 & 1 & 3.84 & 0.17 & $\mathrm{H}_{\mathrm{O}}:$ Accept \\
\hline 5 & BHEL & 2.79 & 1 & 3.84 & 0.10 & $\mathrm{H}_{\mathrm{O}}:$ Accept \\
\hline 6 & CIPLA Ltd & 8.05 & 1 & 3.84 & 0.01 & $\mathrm{H}_{\mathrm{O}}:$ Reject \\
\hline 7 & Grasim Industries Ltd & 10.50 & 1 & 3.84 & 0.00 & $\mathrm{H}_{\mathrm{O}}:$ Reject \\
\hline 8 & HDFC Bank Ltd & 5.58 & 1 & 3.84 & 0.02 & $\mathrm{H}_{\mathrm{O}}:$ Reject \\
\hline 9 & Hero Honda Motors Ltd & 8.39 & 1 & 3.84 & 0.00 & $\mathrm{H}_{\mathrm{O}}:$ Reject \\
\hline 10 & $\begin{array}{l}\text { Hindustan Petroleum } \\
\text { Corporation Ltd }\end{array}$ & 7.63 & 1 & 3.84 & 0.01 & $\mathrm{H}_{\mathrm{O}}:$ Reject \\
\hline 11 & ICICI Bank Ltd & 12.62 & 1 & 3.84 & 0.00 & $\mathrm{H}_{\mathrm{O}}:$ Reject \\
\hline 12 & Infosys Technologies Ltd & 6.06 & 1 & 3.84 & 0.01 & $\mathrm{H}_{\mathrm{O}}:$ Reject \\
\hline 13 & ITC LTD & 1.21 & 1 & 3.84 & 0.27 & $\mathrm{H}_{\mathrm{O}}$ :Accept \\
\hline 14 & M\&M LTD & 8.34 & 1 & 3.84 & 0.00 & $\mathrm{H}_{\mathrm{O}}:$ Reject \\
\hline 15 & ONCG LTD & 3.31 & 1 & 3.84 & 0.07 & $\mathrm{H}_{\mathrm{O}}$ :Accept \\
\hline 16 & Ranbaxy Laboratories Ltd & 11.71 & 1 & 3.84 & 0.00 & $\mathrm{H}_{\mathrm{O}}:$ Reject \\
\hline 17 & Reliance Industries Ltd & 7.06 & 1 & 3.84 & 0.01 & $\mathrm{H}_{\mathrm{O}}:$ Reject \\
\hline 18 & $\begin{array}{l}\text { Sat yam Computer Services } \\
\text { Ltd }\end{array}$ & 8.60 & 1 & 3.84 & 0.00 & $\mathrm{H}_{\mathrm{O}}:$ Reject \\
\hline 19 & Wipro Ltd & 22.49 & 1 & 3.84 & 0.00 & $\mathrm{H}_{\mathrm{O}}:$ Reject \\
\hline
\end{tabular}

Source: Computed

Values of chi-square to test whether there is existence of inter-month, effect on returns of companies listed at BSE. As per this analysis, there is presence of inter-month effect on returns of Bata India limited, ITC limited, ONCG limited, since calculated values of chi-square for these companies are lessen than the table values of chi-squares. For the remaining companies, there is no inter-month effect on returns of the companies.

\section{Results of $\mathbf{H}-$ Test}

A non-parametric Kruskall - wallis test is applied in a place of a conventionally used parametric oneway analysis of variance. It is felt that the kruskall-wallis test is an appropriate one for the data typified of nonnormality, heteroscedastic variance like the security return. Since the result of the normality test indicates that the distributions of the returns are non-normal, we use the non-parametric test, the Kruskal-Wallis to check the results for equlity of mean returns.

The Kruskal-Wallis statistic is as follows:

$\mathrm{H}=\left[\frac{12}{N(N+1)} x \sum_{j=1}^{5} \frac{R j^{2}}{n j}\right]-3(N+1)$

Where: $\mathrm{k}=$ number of samples;

$\mathrm{Nj}=$ number of values in jth sample;

$\mathrm{N}=\sum \mathrm{nj}=$ total number of values;

$\mathrm{Rj}=$ sum of ranks in the sample

When $\mathrm{N}$ values are ranked together (the statistic is approximately Chi-square distributed degrees of freedom equal to k-1). The null hypothesis tested is that there are no differences in the mean daily returns across the weekdays. If the computed ' $H$ ' is greater than the critical value, the null hypothesis cannot be accepted. Conversely, if the computed ' $\mathrm{H}$ ' value is less than the critical value, the alternate hypothesis cannot be accepted.

Pattern of Seasonality is determined by using pair-wise multiple comparison procedure, we can indirectly test which pair shows significant deviations from one another and uncover the general pattern of high - low tendencies in the data. The test procedure relies on the Kruskall - Wallis rank sum $R_{j}$. The data in the rank - day matrix prepared for ' $\mathrm{H}$ ' test is used for this purpose. For a given overall significance level of $\alpha$ decide $\tau_{\mu} \neq \tau_{\mathrm{v}}$

$\mathrm{R}=\left|R_{\mu}-R_{\mathrm{v}}\right| \geq Z[\alpha / K(K-1)][N(N+1) / 12]^{1 / 2}\left[\frac{1}{n_{\mu}}+\frac{1}{n_{\mathrm{v}}}\right] 1 / 2$

Where: $\mu=1,2 \ldots . . \mathrm{k}-1$

$\mathrm{V}=\mu+1 \ldots \mathrm{k}$

$\mathrm{N}=$ total number of daily/weekly means

$\mathrm{n}=$ number of daily /weekly means in the $\mu$ the and vth column

$\mathrm{R}=$ average rank sum of the $\mu \mathrm{th}$ and ith colums 
$\mathrm{Z}(\alpha / \mathrm{K}(\mathrm{K}+1)=$ the upper percentage point of the unit normal distribution for a given value.

The Index returns series and stock returns series of sample companies is tested by using " $\mathrm{H}$ " test and the result is given in Table 9

Ho: There is no difference in the mean returns across the days of the week

H1: There is a difference in the mean returns across the days of the week

The computed ' $\mathrm{H}$ ' value is lower than this critical value for all the BSE and NSE indices, and for selected sample companies also (Table 10) except Hindustan Petroleum Corporation listed BSE, Bata India Ltd listed at NSE and ONGC listed both BSE and NSE at $5 \%$ level of significance with 4 degrees of freedom. So the null hypothesis is accepted. Hence, there is no difference between the mean return across the days of the week except Hindustan Petroleum Corporation and ONGC.

Further h-test is used to test difference in the mean returns across the months of the year with following hypothesis.

Ho: There is no difference in the mean returns across the months of the year

$\mathrm{H} 1$ : There is a difference in the mean returns across the months of the year

The computed ' $\mathrm{H}$ ' value is lower than this critical value for all the BSE and NSE indices and for selected sample companies at $5 \%$ level of significance with 11 degrees of freedom. So the null hypothesis is accepted. Hence, there is no difference between the mean return across the months of the year except Hindustan Petroleum Corporation and ONGC listed at BSE and Hindustan Petroleum Corporation ltd listed at NSE. This provides evidence as to the presence of regularity in common stock return during the study period. Having identified regularity in equity returns, a further enquiry is desirable to uncover the pattern of seasonality.

\begin{tabular}{|c|c|c|c|c|c|c|c|c|c|c|c|c|c|c|c|c|c|c|c|}
\hline \multicolumn{20}{|c|}{$\begin{array}{c}\text { Table }-9 \mathrm{H}-\text { Test } \\
B S E D A I L Y\end{array}$} \\
\hline & $\begin{array}{l}\text { SEN } \\
\text { SEX }\end{array}$ & $\begin{array}{l}\text { ACC } \\
\text { Ltd }\end{array}$ & $\begin{array}{l}\text { Ashok } \\
\text { Leylan } \\
\text { d Ltd }\end{array}$ & $\begin{array}{c}\text { Bata } \\
\text { India } \\
\text { Ltd }\end{array}$ & BHEL & $\begin{array}{l}\text { CIPL } \\
\text { A Ltdd }\end{array}$ & $\begin{array}{c}\text { Grasi } \\
m \\
\text { Indus } \\
\text { tries } \\
\text { Ltd }\end{array}$ & $\begin{array}{c}\text { HDFC } \\
\text { Bank } \\
\text { Ltd }\end{array}$ & $\begin{array}{c}\text { Hero } \\
\text { Hond } \\
\text { a } \\
\text { Motor } \\
s\end{array}$ & $\begin{array}{c}\text { Hindusta } \\
\text { n } \\
\text { Petroleu } \\
\text { m } \\
\text { Corpora } \\
\text { tion }\end{array}$ & $\begin{array}{c}\text { ICICI } \\
\text { Bank } \\
\text { Ltd }\end{array}$ & $\begin{array}{l}\text { Infosys } \\
\text { Technolog } \\
\text { ies Ltd }\end{array}$ & $\begin{array}{l}\text { ITC } \\
\text { LTD }\end{array}$ & $\begin{array}{l}\text { M\&M } \\
\text { LTD }\end{array}$ & $\begin{array}{c}\text { ONG } \\
\text { C }\end{array}$ & $\begin{array}{c}\text { Ranb } \\
\text { axy }\end{array}$ & $\begin{array}{c}\text { Relianc } \\
\text { e }\end{array}$ & $\begin{array}{c}\text { Satya } \\
\text { m }\end{array}$ & $\begin{array}{c}\text { Wipr } \\
0\end{array}$ \\
\hline \multirow{5}{*}{$\begin{array}{l}\text { Mean } \\
\text { rank }\end{array}$} & 1232 & 1238 & 1297 & 1296.9 & 1272.9 & 1252.0 & $\begin{array}{c}1215 . \\
6\end{array}$ & 1251.6 & 1242.8 & 1255.1 & 1270.1 & 1275.8 & 1245.1 & 1264.5 & 1289.4 & 1226.1 & 1236.6 & $\begin{array}{c}1208 . \\
0\end{array}$ & $\begin{array}{c}1218 . \\
5\end{array}$ \\
\hline & 1245 & 1203 & 1183 & 1191 & 1232.5 & 1232.1 & $\begin{array}{c}1240 . \\
0\end{array}$ & 1187.6 & 1233.3 & 1324.8 & 1255.5 & 1280.8 & 1273.8 & 1219.1 & 1179.5 & 1242.7 & 1268.1 & $\begin{array}{c}1233 . \\
2\end{array}$ & $\begin{array}{c}1247 . \\
7\end{array}$ \\
\hline & 1246 & 1269 & 1225 & 1278 & 1244.7 & 1205.3 & $\begin{array}{c}1228 . \\
5\end{array}$ & 1233.5 & 1226.2 & 1229.9 & 1220.2 & 1243.2 & 1237.0 & 1237.9 & 1303.3 & 1232.3 & 1218.9 & $\begin{array}{c}1263 . \\
7\end{array}$ & $\begin{array}{c}1282 . \\
5\end{array}$ \\
\hline & 1235 & 1225.9 & 1275.9 & 1204.8 & 1246.6 & 1262.4 & $\begin{array}{c}1253 . \\
2\end{array}$ & 1287.2 & 1224.3 & 1209.9 & 1216.8 & 1187.9 & 1217.2 & 1233.9 & 1230.8 & 1245.4 & 1261.7 & $\begin{array}{c}1256 . \\
8\end{array}$ & $\begin{array}{c}1244 . \\
9\end{array}$ \\
\hline & 1254 & 1276.9 & 1230.7 & 1241.8 & 1215.5 & 1261.2 & $\begin{array}{c}1275 . \\
8\end{array}$ & 1252.8 & 1286.8 & 1192.0 & 1250.1 & 1224.5 & 1236.9 & 1257.3 & 1208.7 & 1266.3 & 1227.1 & $\begin{array}{c}1250 . \\
9\end{array}$ & $\begin{array}{c}1218 . \\
4\end{array}$ \\
\hline $\begin{array}{c}\text { Chi } \\
\text { square }\end{array}$ & 0.31 & 3.63 & 7.81 & 8.01 & 1.70 & 2.25 & 2.06 & 5.11 & 2.53 & 10.33 & 2.08 & 5.69 & 1.64 & 1.30 & 10.79 & 0.91 & 1.79 & 1.94 & 2.70 \\
\hline $\begin{array}{l}\text { Degrees } \\
\text { of } \\
\text { freedom }\end{array}$ & 4 & 4 & 4 & 4 & 4 & 4 & 4 & 4 & 4 & 4 & 4 & 4 & 4 & 4 & 4 & 4 & 4 & 4 & 4 \\
\hline $\begin{array}{c}\text { Asymp.si } \\
\mathrm{g}\end{array}$ & 0.99 & 0.46 & 0.10 & 0.09 & 0.79 & 0.69 & 0.72 & 0.28 & 0.64 & 0.04 & 0.72 & 0.22 & 0.80 & 0.86 & 0.03 & 0.92 & 0.77 & 0.75 & 0.61 \\
\hline $\begin{array}{c}\text { Inference } \\
\mathrm{s}\end{array}$ & $\begin{array}{c}\mathrm{H}_{\circ} \\
\text { Acce } \\
\mathrm{pt}\end{array}$ & $\begin{array}{l}\mathrm{H}_{\circ} \\
\text { Accept }\end{array}$ & $\begin{array}{l}\mathrm{H}_{\circ} \\
\text { Accept }\end{array}$ & $\begin{array}{l}\mathrm{H}_{\circ} \\
\text { Accept }\end{array}$ & $\begin{array}{c}\mathrm{H}_{\circ} \\
\text { Accept }\end{array}$ & $\begin{array}{c}\mathrm{H}_{\circ} \\
\text { Accep } \\
\mathrm{t}\end{array}$ & $\begin{array}{c}\mathrm{H}_{\circ} \\
\text { Accep } \\
\mathrm{t}\end{array}$ & $\begin{array}{l}\mathrm{H}_{\circ} \\
\text { Accept }\end{array}$ & $\begin{array}{c}\mathrm{H}_{\circ} \\
\text { Accep } \\
\mathrm{t}\end{array}$ & $\begin{array}{c}\mathrm{H}_{\circ} \\
\text { Reject }\end{array}$ & $\begin{array}{l}\mathrm{H}_{\circ} \\
\text { Accept }\end{array}$ & $\begin{array}{c}\mathrm{H}_{\circ} \\
\text { Accept }\end{array}$ & $\begin{array}{l}\mathrm{H}_{\circ} \\
\text { Accept }\end{array}$ & $\begin{array}{c}\mathrm{H}_{\circ} \\
\text { Accep } \\
\mathrm{t}\end{array}$ & $\begin{array}{c}\mathrm{H}_{\circ} \\
\text { Reject }\end{array}$ & $\begin{array}{c}\mathrm{H}_{\circ} \\
\text { Accep } \\
\mathrm{t}\end{array}$ & $\begin{array}{c}\mathrm{H}_{\circ} \\
\text { Accept }\end{array}$ & $\begin{array}{c}\mathrm{H}_{\circ} \\
\text { Accep } \\
\mathrm{t}\end{array}$ & $\begin{array}{c}\mathrm{H}_{\circ} \\
\text { Acce } \\
\text { pt }\end{array}$ \\
\hline
\end{tabular}

\begin{tabular}{|c|c|c|c|c|c|c|c|c|c|c|c|c|c|c|c|c|c|c|c|}
\hline & $\begin{array}{c}\text { SENSE } \\
\mathrm{x}\end{array}$ & $\begin{array}{c}\text { ACC } \\
\text { Ltd }\end{array}$ & $\begin{array}{c}\text { Ashok } \\
\text { Leylan } \\
\text { d Ltd }\end{array}$ & $\begin{array}{c}\text { Bata } \\
\text { India } \\
\text { Ltd }\end{array}$ & $\begin{array}{c}\text { BHE } \\
\text { L }\end{array}$ & $\begin{array}{l}\text { CIPL } \\
\text { A Ltd }\end{array}$ & $\begin{array}{c}\text { Grasim } \\
\text { Industri } \\
\text { es Ltd }\end{array}$ & $\begin{array}{c}\text { HDF } \\
\text { C } \\
\text { Bank } \\
\text { Ltd }\end{array}$ & $\begin{array}{c}\text { Hero } \\
\text { Hond } \\
\text { a } \\
\text { Moto } \\
\text { rs }\end{array}$ & $\begin{array}{c}\text { Hindusta } \\
\text { n } \\
\text { Petroleum } \\
\text { Corporati } \\
\text { on }\end{array}$ & $\begin{array}{c}\text { ICIC } \\
\text { I } \\
\text { Bank } \\
\text { Ltd }\end{array}$ & $\begin{array}{c}\text { Infos } \\
\text { ys } \\
\text { Tech. } \\
\text { Ltd }\end{array}$ & $\begin{array}{c}\text { ITC } \\
\text { LTD }\end{array}$ & $\begin{array}{c}\text { M\& } \\
\text { M } \\
\text { LTD }\end{array}$ & $\begin{array}{c}\text { ONG } \\
\text { C }\end{array}$ & $\begin{array}{c}\text { Ranba } \\
\mathbf{x y}\end{array}$ & $\begin{array}{c}\text { Relian } \\
\text { ce }\end{array}$ & $\begin{array}{c}\text { Satya } \\
\mathrm{m}\end{array}$ & $\begin{array}{c}\text { Wipr } \\
0\end{array}$ \\
\hline \multirow{12}{*}{$\begin{array}{l}\text { Mean } \\
\text { rank }\end{array}$} & 53.30 & 51.80 & 82.60 & 71.75 & 52.00 & 50.00 & 69.80 & 61.15 & 62.20 & 74.00 & 53.65 & 51.80 & 70.90 & 49.80 & 55.90 & 60.50 & 61.80 & 53.20 & 52.60 \\
\hline & 54.40 & 54.60 & 56.60 & 56.20 & 56.30 & 47.20 & 40.20 & 51.10 & 49.50 & 41.60 & 55.70 & 54.60 & 46.90 & 48.60 & 62.90 & 58.60 & 58.90 & 59.80 & 59.40 \\
\hline & 63.60 & 68.70 & 50.90 & 53.20 & 69.50 & 61.30 & 63.10 & 55.50 & 67.50 & 62.30 & 59.50 & 68.70 & 66.50 & 62.20 & 61.50 & 56.70 & 59.40 & 70.60 & 59.30 \\
\hline & 66.00 & 48.10 & 51.60 & 56.00 & \begin{tabular}{|l|}
63.40 \\
\end{tabular} & 69.50 & 69.90 & 71.40 & 63.00 & 68.10 & 61.10 & 48.10 & 54.40 & 63.40 & 73.00 & 65.10 & 60.70 & 49.30 & 50.40 \\
\hline & 72.80 & 79.80 & 77.20 & 80.80 & 77.10 & 80.00 & 76.00 & 57.90 & 62.80 & 76.90 & 53.35 & 79.80 & 74.00 & 57.90 & 60.00 & 91.20 & 65.70 & 63.40 & 74.40 \\
\hline & 57.20 & 57.80 & 51.25 & 51.90 & 48.30 & 70.00 & 53.00 & 71.60 & 65.00 & 49.80 & 62.80 & 57.80 & 38.10 & 44.50 & 50.80 & 58.40 & 72.90 & 46.60 & 47.80 \\
\hline & 53.30 & 73.10 & 54.60 & 48.60 & \begin{tabular}{|l|l|}
58.40 \\
\end{tabular} & 47.30 & 54.60 & 50.50 & 49.10 & 54.70 & 59.10 & 73.10 & 46.30 & 64.40 & 55.10 & 39.00 & 52.10 & 67.30 & 56.20 \\
\hline & 80.80 & 75.80 & 66.75 & 75.35 & 69.60 & 76.80 & 65.90 & 67.30 & 76.10 & 68.20 & 66.00 & 75.80 & 76.60 & 82.50 & 62.00 & 74.90 & 61.30 & 83.20 & 89.30 \\
\hline & 76.70 & 65.20 & 73.70 & 82.20 & 58.60 & 69.90 & 69.90 & 75.40 & 67.60 & 68.60 & 79.30 & 65.20 & 71.70 & 78.20 & 62.40 & 67.30 & 76.80 & 71.30 & 71.50 \\
\hline & 52.90 & 46.10 & 45.60 & 45.00 & 52.30 & 39.30 & 56.80 & 66.00 & 59.60 & 56.30 & 61.50 & 46.10 & 65.90 & 69.00 & 61.90 & 49.00 & 54.10 & 57.50 & 60.90 \\
\hline & 53.50 & 52.70 & 75.50 & 50.40 & 71.30 & 47.80 & 54.40 & 47.15 & 54.80 & 53.50 & 56.20 & 52.70 & 67.80 & 60.70 & 52.20 & 54.70 & 53.30 & 53.30 & 54.00 \\
\hline & 41.50 & 52.30 & 39.70 & 54.60 & 49.20 & 66.90 & 52.40 & 51.00 & 48.80 & 52.00 & 57.80 & 52.30 & 46.90 & 44.80 & 68.30 & 50.60 & 49.00 & 50.50 & 50.20 \\
\hline $\begin{array}{l}\text { Chi } \\
\text { square }\end{array}$ & 12.28 & 12.08 & 17.91 & 15.82 & 8.32 & 16.69 & 9.58 & 8.51 & 6.60 & 10.59 & 4.46 & 12.08 & 15.74 & 13.73 & 3.68 & 16.25 & 6.18 & 10.92 & 13.55 \\
\hline $\begin{array}{l}\text { Degrees } \\
\text { of } \\
\text { freedom }\end{array}$ & 11 & 11 & 11 & 11 & 11 & 11 & 11 & 11 & 11 & 11 & 11 & 11 & 11 & 11 & 11 & 11 & 11 & 11 & 11 \\
\hline $\begin{array}{l}\text { Asymp.s } \\
\text { ig }\end{array}$ & 0.34 & 0.36 & 0.08 & 0.15 & 0.69 & 0.12 & 0.57 & 0.67 & 0.83 & 0.48 & 0.95 & 0.36 & 0.15 & 0.25 & 0.98 & 0.13 & 0.86 & 0.45 & 0.26 \\
\hline $\begin{array}{l}\text { Inferenc } \\
\text { es }\end{array}$ & $\begin{array}{c}\mathrm{H}_{0} \\
\text { Accept }\end{array}$ & $\begin{array}{c}\mathrm{H}_{0} \\
\text { Acce } \\
\mathrm{pt}\end{array}$ & $\begin{array}{c}\mathrm{H}_{0} \\
\text { Accept }\end{array}$ & $\begin{array}{c}\mathrm{H}_{0} \\
\text { Acce } \\
\mathrm{pt}\end{array}$ & $\begin{array}{c}\mathrm{H}_{0} \\
\text { Acce } \\
\mathrm{pt}\end{array}$ & $\begin{array}{c}\mathrm{H}_{0} \\
\text { Accep } \\
\mathrm{t}\end{array}$ & $\begin{array}{c}\mathrm{H}_{0} \\
\text { Accept }\end{array}$ & $\begin{array}{c}\mathrm{H}_{0} \\
\text { Acce } \\
\mathrm{pt}\end{array}$ & $\begin{array}{c}\mathrm{H}_{0} \\
\text { Accep } \\
\mathrm{t}\end{array}$ & $\begin{array}{c}\mathrm{H}_{0} \\
\text { Accept }\end{array}$ & $\begin{array}{c}\mathrm{H}_{0} \\
\text { Acce } \\
\mathrm{pt}\end{array}$ & $\begin{array}{c}\mathrm{H}_{0} \\
\text { Accep } \\
\mathrm{t}\end{array}$ & $\begin{array}{c}\mathrm{H}_{0} \\
\text { Acce } \\
\mathrm{pt}\end{array}$ & $\begin{array}{c}\mathrm{H}_{0} \\
\text { Acce } \\
\mathrm{pt}\end{array}$ & $\begin{array}{c}\mathrm{H}_{0} \\
\text { Acce } \\
\mathrm{pt}\end{array}$ & $\begin{array}{c}\mathrm{H}_{0} \\
\text { Accept }\end{array}$ & $\begin{array}{c}\mathrm{H}_{0} \\
\text { Accept }\end{array}$ & $\begin{array}{c}\mathrm{H}_{0} \\
\text { Accep } \\
\mathrm{t}\end{array}$ & $\begin{array}{c}\mathrm{H}_{0} \\
\text { Acce } \\
\mathrm{pt}\end{array}$ \\
\hline
\end{tabular}

Source: Computed data 


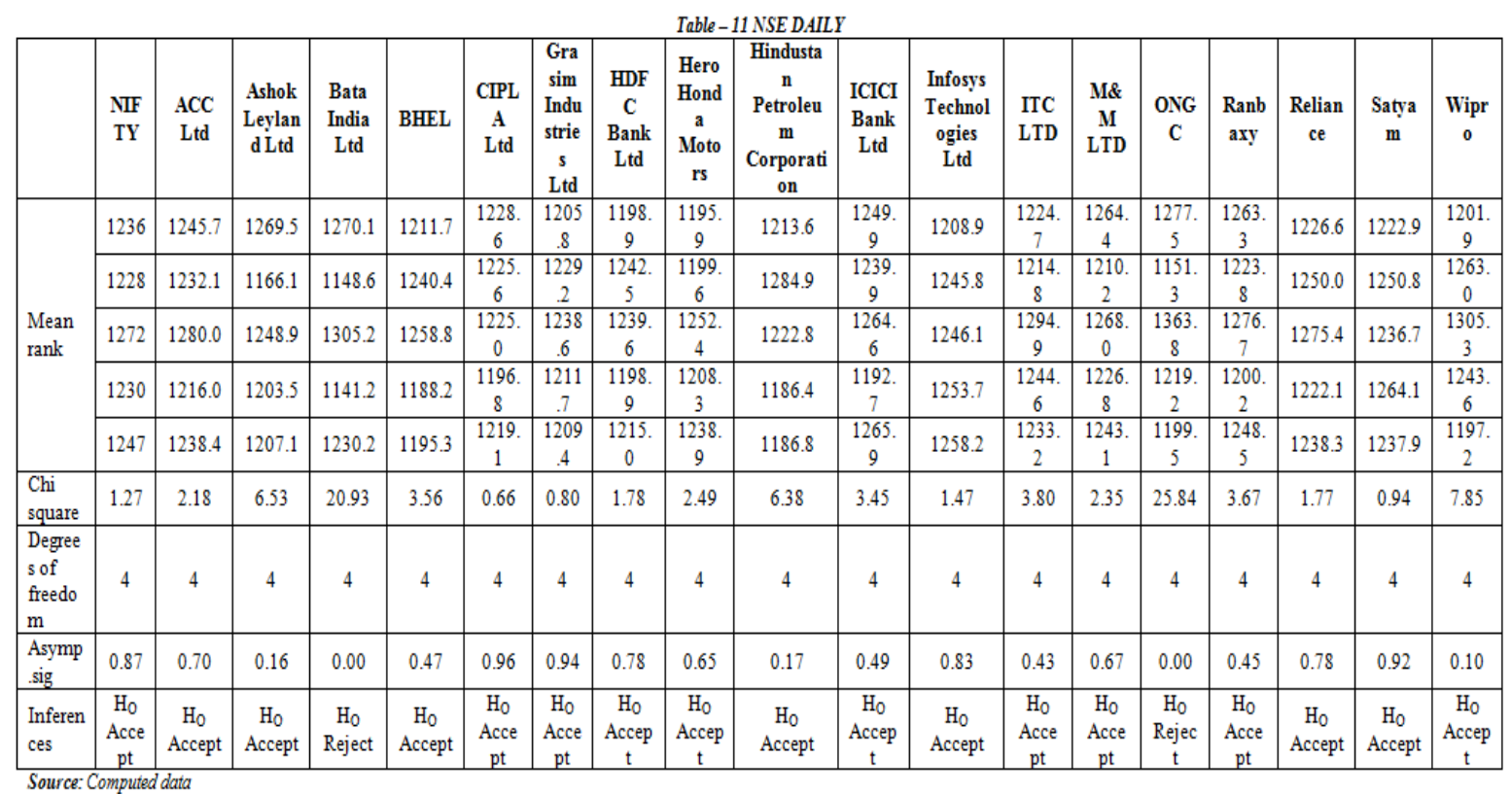

\section{Table - 12 NSE MONTHLY} Table -12NSE MONTHLY

\begin{tabular}{|c|c|c|c|c|c|c|c|c|c|c|c|c|c|c|c|c|c|c|c|}
\hline & $\begin{array}{c}\text { NIFT } \\
Y\end{array}$ & $\begin{array}{c}\mathrm{ACC} \\
\text { Ltd }\end{array}$ & $\begin{array}{l}\text { Ashok } \\
\text { Leylan } \\
\text { d Ltd }\end{array}$ & $\begin{array}{c}\text { Bata } \\
\text { India } \\
\text { Ltd }\end{array}$ & $\begin{array}{c}\text { BHE } \\
\text { L }\end{array}$ & $\begin{array}{l}\text { CIPL } \\
\text { A Ltd }\end{array}$ & $\begin{array}{c}\text { Grasim } \\
\text { Industrie } \\
\text { s Ltd }\end{array}$ & $\begin{array}{c}\mathrm{HDF} \\
\mathrm{C} \\
\text { Bank } \\
\text { Ltd } \\
\end{array}$ & $\begin{array}{c}\text { Hero } \\
\text { Honda } \\
\text { Motor } \\
\text { s } \\
\end{array}$ & $\begin{array}{c}\text { Hindustan } \\
\text { Petroleum } \\
\text { Corporatio } \\
\text { n }\end{array}$ & $\begin{array}{c}\text { ICICI } \\
\text { Bank } \\
\text { Ltd }\end{array}$ & $\begin{array}{c}\text { Infosy } \\
\text { s Tech } \\
\text { Ltd }\end{array}$ & $\begin{array}{l}\text { ITC } \\
\text { LTD }\end{array}$ & $\begin{array}{c}\text { M\& } \\
\text { M } \\
\text { LTD }\end{array}$ & $\begin{array}{c}\text { ONG } \\
\mathrm{C}\end{array}$ & $\begin{array}{c}\text { Ranbax } \\
y\end{array}$ & $\begin{array}{c}\text { Relianc } \\
\text { e }\end{array}$ & $\begin{array}{c}\text { Satya } \\
\text { m }\end{array}$ & $\begin{array}{c}\text { Wipr } \\
0\end{array}$ \\
\hline \multirow{12}{*}{$\begin{array}{l}\text { Mean } \\
\text { rank }\end{array}$} & 47.20 & 60.50 & 68.30 & 69.90 & 56.00 & 58.70 & 60.90 & 52.90 & 41.40 & 50.70 & 46.80 & 45.60 & 65.90 & 40.70 & 62.20 & 64.40 & 65.00 & 52.70 & 46.50 \\
\hline & 51.40 & 43.90 & 71.50 & 58.30 & 56.10 & 37.20 & 54.80 & 64.10 & 64.10 & 63.30 & 53.30 & 56.30 & 56.70 & 55.10 & 60.50 & 61.50 & 60.60 & 59.60 & 60.90 \\
\hline & 55.10 & 39.00 & 55.50 & 52.30 & 48.30 & 56.70 & 50.30 & 46.90 & 59.90 & 45.20 & 56.10 & 61.50 & 48.20 & 50.10 & 56.10 & 56.50 & 56.10 & 56.30 & 53.70 \\
\hline & 69.10 & 78.30 & 48.40 & 62.60 & 79.70 & 72.90 & 71.60 & 67.50 & 66.20 & 50.50 & 54.00 & 47.10 & 78.90 & 67.90 & 72.90 & 65.20 & 58.00 & 65.40 & 51.40 \\
\hline & 63.30 & 73.00 & 69.40 & 63.40 & 63.80 & 74.10 & 74.50 & 60.20 & 59.30 & 53.90 & 58.20 & 62.10 & 57.60 & 61.30 & 69.40 & 73.40 & 57.40 & 53.20 & 64.70 \\
\hline & 71.10 & 59.70 & 56.90 & 71.60 & 61.50 & 80.00 & 64.80 & 64.30 & 70.00 & 67.40 & 60.50 & 76.60 & 59.70 & 49.40 & 60.60 & 85.10 & 75.60 & 58.20 & 59.90 \\
\hline & 59.90 & 51.60 & 51.80 & 48.30 & 59.10 & 61.10 & 52.20 & 65.40 & 57.10 & 43.70 & 58.20 & 67.70 & 38.70 & 57.30 & 54.10 & 47.00 & 61.80 & 57.70 & 53.90 \\
\hline & 68.20 & 70.80 & 60.80 & 70.30 & 69.70 & 51.70 & 63.40 & 55.30 & 61.00 & 77.30 & 72.00 & 81.50 & 58.90 & 75.70 & 53.80 & 45.20 & 59.10 & 74.30 & 74.60 \\
\hline & 84.90 & 86.80 & 76.60 & 85.60 & 62.30 & 82.90 & 66.10 & 70.40 & 71.40 & 91.70 & 82.70 & 76.00 & 81.10 & 78.20 & 60.40 & 70.40 & 68.80 & 77.90 & 94.00 \\
\hline & 62.60 & 62.80 & 59.00 & 57.60 & 57.50 & 56.10 & 70.30 & 74.10 & 70.10 & 75.90 & 70.10 & 55.10 & 78.20 & 75.00 & 63.70 & 55.20 & 65.60 & 63.30 & 63.30 \\
\hline & 57.00 & 59.20 & 62.80 & 40.10 & 74.70 & 46.90 & 56.70 & 62.50 & 55.10 & 65.90 & 65.00 & 45.00 & 56.80 & 68.00 & 52.00 & 57.90 & 59.30 & 57.90 & 59.40 \\
\hline & 35.80 & 40.40 & 45.40 & 46.00 & 37.30 & 47.70 & 40.40 & 42.40 & 50.40 & 40.50 & 49.10 & 51.50 & 45.30 & 47.30 & 60.30 & 44.20 & 38.70 & 49.50 & 43.70 \\
\hline $\begin{array}{c}\text { Chi } \\
\text { square }\end{array}$ & 14.66 & 20.66 & 8.40 & 14.93 & 11.63 & 18.41 & 9.07 & 8.14 & 7.08 & 22.59 & 9.75 & 14.59 & 16.55 & 14.08 & 3.50 & 13.56 & 7.10 & 6.60 & 16.52 \\
\hline $\begin{array}{c}\text { Degrees } \\
\text { of } \\
\text { freedom }\end{array}$ & 11 & 11 & 11 & 11 & 11 & 11 & 11 & 11 & 11 & 11 & 11 & 11 & 11 & 11 & 11 & 11 & 11 & 11 & 11 \\
\hline $\begin{array}{c}\text { Asymp.si } \\
\mathrm{g}\end{array}$ & 0.20 & 0.04 & 0.68 & 0.19 & 0.39 & 0.07 & 0.62 & 0.70 & 0.79 & 0.02 & 0.55 & 0.20 & 0.12 & 0.23 & 0.98 & 0.26 & 0.79 & 0.83 & 0.12 \\
\hline $\begin{array}{c}\text { Inference } \\
s\end{array}$ & $\begin{array}{c}\mathrm{H}_{0} \\
\text { Accept }\end{array}$ & $\begin{array}{c}\mathrm{H}_{0} \\
\text { Rejec } \\
\mathrm{t}\end{array}$ & $\begin{array}{c}\mathrm{H}_{0} \\
\text { Accept }\end{array}$ & $\begin{array}{c}\mathrm{H}_{0} \\
\text { Accep } \\
\mathrm{t}\end{array}$ & $\begin{array}{c}\mathrm{H}_{0} \\
\text { Accep } \\
\mathrm{t}\end{array}$ & $\begin{array}{c}\mathrm{H}_{0} \\
\text { Accept }\end{array}$ & $\begin{array}{c}\mathrm{H}_{0} \\
\text { Accept }\end{array}$ & $\begin{array}{c}\mathrm{H}_{0} \\
\text { Accep } \\
\mathrm{t}\end{array}$ & $\begin{array}{c}\mathrm{H}_{0} \\
\text { Accept }\end{array}$ & $\begin{array}{c}\mathrm{H}_{0} \\
\text { Reject }\end{array}$ & $\begin{array}{c}\mathrm{H}_{0} \\
\text { Accep } \\
\mathrm{t}\end{array}$ & $\begin{array}{c}\mathrm{H}_{0} \\
\text { Accept }\end{array}$ & $\begin{array}{c}\mathrm{H}_{0} \\
\text { Accep } \\
\mathrm{t}\end{array}$ & $\begin{array}{c}\mathrm{H}_{0} \\
\text { Accep } \\
\mathrm{t}\end{array}$ & $\begin{array}{c}\mathrm{H}_{0} \\
\text { Accept }\end{array}$ & $\begin{array}{c}\mathrm{H}_{0} \\
\text { Accept }\end{array}$ & $\begin{array}{c}\mathrm{H}_{0} \\
\text { Accept }\end{array}$ & $\begin{array}{c}\mathrm{H}_{0} \\
\text { Accept }\end{array}$ & $\begin{array}{c}\mathrm{H}_{0} \\
\text { Accep } \\
\mathrm{t}\end{array}$ \\
\hline
\end{tabular}

Source: Computed data

Since The Levene Statistic $\rho$ value $=$ sig is greater than $\alpha=0.01$, so we fail to reject the null hypothesis that the variance are all equal. Since the variances appear to be equal, we may continue with ANOVA.

In order to test the equality of means of daily stock returns and market returns of week days, the null and alternative hypotheses are $; \mathrm{H}_{0}: \mu_{1}=\mu_{2}=\mu_{3}=\mu_{4}=\mu_{5}$ (the means of the stock return and market return for five days are equal) $\mathrm{H}_{1}$ : at least one pair is unequal.It is assumed that stock return and market return obtained are distributed normally with means $\mu_{1}, \mu_{2}, \mu_{3}, \mu_{4}$, and $\mu_{5}$ for five days Monday, Tuesday, Wednesday, Thursday and Friday respectively. Further it is assumed that the standard deviations of the distribution of returns for Monday, Tuesday, Wednesday, Thursday and Friday are equal and constant. This assumption implies that the mean returns may differ on account of trading the shares at different days in the stock exchange, but they do not affect the dispersion of the returns.

The foregoing calculations can be summarized in the Table 13. 
Stock Market Seasonality- Time Varying Volatility In The Emerging Indian Stock Market

\begin{tabular}{|c|c|c|c|c|c|c|c|c|c|c|}
\hline & & ble & ANOS & R SEAS & NALI & TY BSE & AILY & & & \\
\hline Companies & $\begin{array}{c}\text { Levene } \\
\text { Statistics }\end{array}$ & Sing & & $\begin{array}{c}\text { Sum of } \\
\text { squares }\end{array}$ & df & $\begin{array}{l}\text { Mean } \\
\text { square }\end{array}$ & $\mathbf{F}$ & sign & $\mathbf{R}^{2}$ & $\underset{\mathbf{R}^{2}}{\text { Adjust }}$ \\
\hline & 1.561 & 0.182 & $\begin{array}{l}\text { Between } \\
\text { groups }\end{array}$ & 0.040 & 4 & 0.010 & 1.378 & 0.239 & & \\
\hline ACC Ltd & & & $\begin{array}{l}\text { Within } \\
\text { groups }\end{array}$ & 18.086 & 2479 & 0.007 & - & - & 0.002 & 0.001 \\
\hline Ashok & 0.811 & 0.518 & $\begin{array}{l}\text { Between } \\
\text { groups }\end{array}$ & 0.185 & 4 & 0.046 & 1.041 & 0.385 & 0007 & 0000 \\
\hline Leyland Ltd & & & $\begin{array}{l}\text { Within } \\
\text { groups }\end{array}$ & 110.36 & 2479 & 0.045 & - & - & 0.002 & 0.000 \\
\hline Bata India & 0.403 & 0.807 & $\begin{array}{l}\text { Between } \\
\text { groups }\end{array}$ & 0.083 & 4 & 0.021 & 1.002 & 0.405 & & \\
\hline Ltd & & & $\begin{array}{l}\text { Within } \\
\text { groups }\end{array}$ & 51.328 & 2479 & 0.021 & - & - & 0.002 & 0.000 \\
\hline Pholl & 0.065 & 0.992 & $\begin{array}{l}\text { Between } \\
\text { groups }\end{array}$ & 0.005 & 4 & 0.001 & 0.305 & 0.874 & 0000 & 0001 \\
\hline Bnell & & & $\begin{array}{l}\text { Within } \\
\text { groups }\end{array}$ & 10.924 & 2479 & 0.004 & - & - & 0.000 & -0.001 \\
\hline & 0.302 & 0.877 & $\begin{array}{l}\text { Between } \\
\text { groups }\end{array}$ & 0.016 & 4 & 0.004 & 1.319 & 0.261 & 0000 & 0001 \\
\hline Cipla Ltd & & & $\begin{array}{l}\text { Within } \\
\text { groups }\end{array}$ & 7.520 & 2479 & 0.003 & - & - & 0.002 & 0.001 \\
\hline Grasim & 0.950 & 0.434 & $\begin{array}{l}\text { Between } \\
\text { groups }\end{array}$ & 0.096 & 4 & 0.024 & 1.052 & 0.379 & 0000 & 0000 \\
\hline Ltd & & & $\begin{array}{l}\text { Within } \\
\text { groups }\end{array}$ & 56.398 & 2479 & 0.023 & - & - & 0.002 & 0.000 \\
\hline HDFC Bank & 1.031 & 0.389 & $\begin{array}{l}\text { Between } \\
\text { groups }\end{array}$ & 0.021 & 4 & 0.005 & 0.536 & 0.709 & & \\
\hline Ltd & & & $\begin{array}{l}\text { Within } \\
\text { groups }\end{array}$ & 24.219 & 2479 & 0.010 & - & - & 0.001 & -0.001 \\
\hline Hero Honda & 0.780 & 0.538 & $\begin{array}{l}\text { Between } \\
\text { groups }\end{array}$ & 0.025 & 4 & 0.006 & 2.334 & 0.054 & $0 \Omega 04$ & 0000 \\
\hline Motors Ltd & & & $\begin{array}{l}\text { Within } \\
\text { groups }\end{array}$ & 6.733 & 2479 & 0.003 & - & - & 0.004 & 0.002 \\
\hline Hindustan & 2.110 & 0.077 & $\begin{array}{l}\text { Between } \\
\text { groups }\end{array}$ & 0.007 & 4 & 0.002 & 1.612 & 0.168 & & \\
\hline $\begin{array}{l}\text { Petroleum } \\
\text { Corporation }\end{array}$ & & & $\begin{array}{l}\text { Within } \\
\text { groups }\end{array}$ & 2.563 & 2479 & 0.001 & - & - & 0.003 & 0.001 \\
\hline ICICI Bank & 2.574 & 0.036 & $\begin{array}{l}\text { Between } \\
\text { groups }\end{array}$ & 0.001 & 4 & 0.000 & 0.216 & 0.930 & 0000 & - \\
\hline & & & $\begin{array}{l}\text { Within } \\
\text { groups }\end{array}$ & 2.227 & 2479 & 0.001 & - & - & 0.000 & -0.000 \\
\hline Infosys & 1.532 & 0.190 & $\begin{array}{l}\text { Between } \\
\text { groups }\end{array}$ & 0.013 & 4 & 0.003 & 1.803 & 0.125 & 0002 & 0001 \\
\hline Technologies & & & $\begin{array}{l}\text { Within } \\
\text { groups }\end{array}$ & 4.600 & 2479 & 0.002 & - & - & 0.003 & 0.001 \\
\hline ITC Itd & 1.209 & 0.305 & $\begin{array}{l}\text { Between } \\
\text { groups }\end{array}$ & 0.007 & 4 & 0.002 & 0.807 & 0.520 & 0001 & 0000 \\
\hline I1C Ltd & & & $\begin{array}{l}\text { Within } \\
\text { groups }\end{array}$ & 5.288 & 2479 & 0.002 & - & - & 0.001 & 0.000 \\
\hline & 1.283 & 0.274 & $\begin{array}{l}\text { Between } \\
\text { groups }\end{array}$ & 0.129 & 4 & 0.032 & 1.199 & 0.309 & & \\
\hline M\&M Ltd & & & $\begin{array}{l}\text { Within } \\
\text { groups }\end{array}$ & 66.754 & 2479 & 0.027 & - & - & 0.002 & 0.000 \\
\hline & 0.624 & 0.646 & $\begin{array}{l}\text { Between } \\
\text { groups }\end{array}$ & 0.051 & 4 & 0.013 & 1.711 & 0.145 & & \\
\hline ON & & & $\begin{array}{l}\text { Within } \\
\text { groups }\end{array}$ & 18.502 & 2479 & 0.007 & - & - & .003 & 0.001 \\
\hline Ranbaxy & 1.764 & 0.133 & $\begin{array}{l}\text { Between } \\
\text { groups }\end{array}$ & 0.015 & 4 & 0.004 & 1.169 & 0.322 & & \\
\hline Laboratories & & & $\begin{array}{l}\text { Within } \\
\text { groups }\end{array}$ & 7.882 & 2479 & 0.003 & - & - & 0.002 & 0.000 \\
\hline Reliance & 2.226 & 0.064 & Between & 0.008 & 4 & 0.002 & 0.895 & 0.466 & 0.001 & 0.000 \\
\hline
\end{tabular}


Stock Market Seasonality- Time Varying Volatility In The Emerging Indian Stock Market

\begin{tabular}{|c|c|c|c|c|c|c|c|c|c|c|}
\hline \multirow{2}{*}{$\begin{array}{l}\text { Industries } \\
\text { Ltd }\end{array}$} & & & groups & & & & & & & \\
\hline & & & $\begin{array}{l}\text { Within } \\
\text { groups }\end{array}$ & 5.880 & 2479 & 0.002 & - & - & & \\
\hline \multirow{2}{*}{$\begin{array}{l}\text { Sathyam } \\
\text { Computers } \\
\text { Services Ltd }\end{array}$} & 0.733 & 0.569 & $\begin{array}{l}\text { Between } \\
\text { groups }\end{array}$ & 0.013 & 4 & 0.003 & 1.229 & 0.296 & \multirow{2}{*}{0.002} & \multirow{2}{*}{0.000} \\
\hline & & & $\begin{array}{l}\text { Within } \\
\text { groups }\end{array}$ & 6.675 & 2479 & 0.003 & - & - & & \\
\hline \multirow{2}{*}{ Wipro Ltd } & 0.335 & 0.854 & $\begin{array}{l}\text { Between } \\
\text { groups }\end{array}$ & 0.103 & 4 & 0.026 & 0.943 & 0.438 & \multirow{2}{*}{0.002} & \multirow{2}{*}{0.000} \\
\hline & & & $\begin{array}{l}\text { Within } \\
\text { groups }\end{array}$ & 67.498 & 2479 & 0.027 & - & - & & \\
\hline \multirow{2}{*}{ Sensex } & 0.637 & 0.636 & $\begin{array}{l}\text { Between } \\
\text { groups }\end{array}$ & 0.047 & 4 & 0.012 & 0.860 & 0.487 & \multirow{2}{*}{0.001} & \multirow{2}{*}{0.000} \\
\hline & & & $\begin{array}{l}\text { Within } \\
\text { groups }\end{array}$ & 34.184 & 2479 & 0.014 & - & - & & \\
\hline
\end{tabular}

Table - 14 ANOVA FOR SEASONALITY NSE DAILY

\begin{tabular}{|c|c|c|c|c|c|c|c|c|}
\hline Companies & & Sum $^{2}$ & df & Mean $^{2}$ & $\mathbf{F}$ & sign & $\mathbf{R}^{2}$ & Adjust R $^{2}$ \\
\hline \multirow[t]{2}{*}{ ACC Ltd } & $\begin{array}{l}\text { Between } \\
\text { groups }\end{array}$ & 0.001 & 4 & 0.000 & 0.192 & 0.943 & \multirow[t]{2}{*}{0.000} & \multirow[t]{2}{*}{-0.001} \\
\hline & Within groups & 2.785 & 2479 & 0.001 & - & - & & \\
\hline \multirow[t]{2}{*}{$\begin{array}{l}\text { Ashok } \\
\text { Leyland Ltd }\end{array}$} & $\begin{array}{l}\text { Between } \\
\text { groups }\end{array}$ & 0.007 & 4 & 0.002 & 1.217 & 0.301 & \multirow[t]{2}{*}{0.002} & \multirow[t]{2}{*}{0.000} \\
\hline & Within groups & 3.402 & 2479 & 0.001 & - & - & & \\
\hline \multirow[t]{2}{*}{$\begin{array}{l}\text { Bata India } \\
\text { Ltd }\end{array}$} & $\begin{array}{l}\text { Between } \\
\text { groups }\end{array}$ & 0.013 & 4 & 0.003 & 2.695 & 0.029 & \multirow[t]{2}{*}{0.004} & \multirow[t]{2}{*}{0.003} \\
\hline & Within groups & 3.037 & 2479 & 0.001 & - & - & & \\
\hline \multirow[t]{2}{*}{ Bhell } & $\begin{array}{l}\text { Between } \\
\text { groups }\end{array}$ & 0.004 & 4 & 0.001 & 1.003 & 0.405 & \multirow[t]{2}{*}{0.002} & \multirow[t]{2}{*}{0.000} \\
\hline & Within groups & 2.412 & 2479 & 0.001 & - & - & & \\
\hline \multirow[t]{2}{*}{ Cipla Ltd } & $\begin{array}{l}\text { Between } \\
\text { groups }\end{array}$ & 0.003 & 4 & 0.001 & 0.536 & 0.709 & \multirow[t]{2}{*}{0.001} & \multirow[t]{2}{*}{-0.001} \\
\hline & Within groups & 2.928 & 2479 & 0.001 & - & - & & \\
\hline \multirow[t]{2}{*}{$\begin{array}{l}\text { Grasim } \\
\text { Industries Ltd }\end{array}$} & $\begin{array}{l}\text { Between } \\
\text { groups }\end{array}$ & 0.002 & 4 & 0.001 & 0.822 & 0.511 & \multirow[t]{2}{*}{0.001} & \multirow[t]{2}{*}{0.000} \\
\hline & Within groups & 1.858 & 2479 & 0.001 & - & - & & \\
\hline \multirow[t]{2}{*}{$\begin{array}{l}\text { HDFC Bank } \\
\text { Ltd }\end{array}$} & $\begin{array}{l}\text { Between } \\
\text { groups }\end{array}$ & 0.003 & 4 & 0.001 & 0.074 & 0.589 & \multirow[t]{2}{*}{0.001} & \multirow[t]{2}{*}{0.000} \\
\hline & Within groups & 2.388 & 2479 & 0.001 & - & - & & \\
\hline \multirow[t]{2}{*}{$\begin{array}{l}\text { Hero Honda } \\
\text { Motors Ltd }\end{array}$} & $\begin{array}{l}\text { Between } \\
\text { groups }\end{array}$ & 0.003 & 4 & 0.001 & 1.052 & 0.379 & \multirow[t]{2}{*}{0.002} & \multirow[t]{2}{*}{0.000} \\
\hline & Within groups & 1.712 & 2479 & 0.001 & - & - & & \\
\hline \multirow{2}{*}{$\begin{array}{l}\text { Hindustan } \\
\text { Petroleum } \\
\text { Corporation } \\
\text { Ltd } \\
\end{array}$} & $\begin{array}{l}\text { Between } \\
\text { groups }\end{array}$ & 0.003 & 4 & 0.001 & 0.819 & 0.513 & \multirow[t]{2}{*}{0.001} & \multirow[t]{2}{*}{0.000} \\
\hline & Within groups & 2.459 & 2479 & 0.001 & - & - & & \\
\hline \multirow[t]{2}{*}{$\begin{array}{l}\text { ICICI Bank } \\
\text { Ltd }\end{array}$} & $\begin{array}{l}\text { Between } \\
\text { groups }\end{array}$ & 0.003 & 4 & 0.001 & 0.732 & 0.570 & \multirow[t]{2}{*}{0.001} & 0.000 \\
\hline & Within groups & 2.741 & 2479 & 0.001 & - & - & & \\
\hline $\begin{array}{l}\text { Infosys } \\
\text { Technologies }\end{array}$ & $\begin{array}{l}\text { Between } \\
\text { groups }\end{array}$ & 0.005 & 4 & 0.001 & 1.011 & 0.400 & 0.002 & 0.000 \\
\hline Ltd & Within groups & 3.246 & 2479 & 0.001 & - & - & & \\
\hline ITC Ltd & $\begin{array}{l}\text { Between } \\
\text { groups }\end{array}$ & 0.001 & 4 & 0.000 & 0.314 & 0.869 & 0.001 & -0.001 \\
\hline & Within groups & 2.186 & 2479 & 0.001 & - & - & & \\
\hline M\&M Ltd & $\begin{array}{l}\text { Between } \\
\text { groups }\end{array}$ & 0.002 & 4 & 0.001 & 0.524 & 0.718 & 0.001 & -0.001 \\
\hline & Within groups & 2.544 & 2479 & 0.001 & - & - & & \\
\hline ONGC Ltd & $\begin{array}{l}\text { Between } \\
\text { groups }\end{array}$ & 0.016 & 4 & 0.004 & 5.241 & 0.000 & 0.008 & 0.007 \\
\hline & Within groups & 1.867 & 2479 & 0.001 & - & - & & \\
\hline $\begin{array}{l}\text { Ranbaxy } \\
\text { Laboratories }\end{array}$ & $\begin{array}{l}\text { Between } \\
\text { groups }\end{array}$ & 0.004 & 4 & 0.001 & 1.069 & 0.370 & 0.002 & 0.000 \\
\hline Ltd & Within groups & 2.185 & 2479 & 0.001 & - & - & & \\
\hline
\end{tabular}


Stock Market Seasonality- Time Varying Volatility In The Emerging Indian Stock Market

\begin{tabular}{|l|l|c|c|c|c|c|c|c|}
\hline \multirow{2}{*}{$\begin{array}{l}\text { Reliance } \\
\text { Industries Ltd }\end{array}$} & $\begin{array}{l}\text { Between } \\
\text { groups }\end{array}$ & 0.001 & 4 & 0.000 & 0.538 & 0.708 & 0.001 & -0.001 \\
\cline { 2 - 7 } & Within groups & 1.677 & 2479 & 0.001 & - & - & & \\
\hline \multirow{2}{*}{$\begin{array}{l}\text { Sathyam } \\
\text { Computers } \\
\text { Services Ltd }\end{array}$} & $\begin{array}{l}\text { Between } \\
\text { groups }\end{array}$ & 0.011 & 4 & 0.003 & 1.133 & 0.339 & 0.002 & 0.000 \\
\cline { 2 - 7 } Within groups & 5.783 & 2479 & 0.002 & - & - & & \\
\hline \multirow{2}{*}{ Nifty } & $\begin{array}{l}\text { Between } \\
\text { groups }\end{array}$ & 0.009 & 4 & 0.002 & 1.279 & 0.276 & 0.002 & 0.000 \\
\cline { 2 - 6 } & Within groups & 4.483 & 2479 & 0.002 & - & - & & \\
\hline & $\begin{array}{l}\text { Between } \\
\text { groups }\end{array}$ & 0.001 & 4 & 0.000 & 0.748 & 0.559 & 0.001 & 0.000 \\
\cline { 2 - 6 } & Within groups & 0.746 & 2479 & 0.000 & - & - & & \\
\hline
\end{tabular}

Table - 15 ANOVA FOR SEASONALITY BSE MONTHLY

\begin{tabular}{|c|c|c|c|c|c|c|c|c|}
\hline Companies & & Sum $^{2}$ & df & Mean $^{2}$ & $\mathbf{F}$ & sign & $\mathbf{R}^{2}$ & Adjust $R^{2}$ \\
\hline \multirow[t]{2}{*}{ ACC Ltd } & Between groups & 0.312 & 11 & 0.028 & 1.274 & 0.249 & \multirow[t]{2}{*}{0.115} & \multirow[t]{2}{*}{0.025} \\
\hline & Within groups & 2.409 & 108 & 0.022 & - & - & & \\
\hline \multirow[t]{2}{*}{ Ashok Leyland Ltd } & Between groups & 0.546 & 11 & 0.050 & 1.185 & 0.306 & \multirow[t]{2}{*}{0.108} & \multirow[t]{2}{*}{0.017} \\
\hline & Within groups & 4.524 & 108 & 0.042 & - & - & & \\
\hline \multirow[t]{2}{*}{ Bata India Ltd } & Between groups & 0.426 & 11 & 0.039 & 1.516 & 0.136 & \multirow[t]{2}{*}{0.134} & \multirow[t]{2}{*}{0.045} \\
\hline & Within groups & 2.759 & 108 & 0.026 & - & - & & \\
\hline \multirow[t]{2}{*}{ Bhell } & Between groups & 0.172 & 11 & 0.016 & 0.887 & 0.555 & \multirow[t]{2}{*}{0.083} & \multirow[t]{2}{*}{-0.011} \\
\hline & Within groups & 1.906 & 108 & 0.018 & - & - & & \\
\hline \multirow[t]{2}{*}{ Cipla Ltd } & Between groups & 0.417 & 11 & 0.038 & 1.455 & 0.159 & \multirow[t]{2}{*}{0.129} & \multirow[t]{2}{*}{0.040} \\
\hline & Within groups & 2.816 & 108 & 0.026 & - & - & & \\
\hline \multirow[t]{2}{*}{ Grasim Industries Ltd } & Between groups & 0.235 & 11 & 0.021 & 0.829 & 0.611 & \multirow[t]{2}{*}{0.078} & \multirow[t]{2}{*}{-0.016} \\
\hline & Within groups & 2.784 & 108 & 0.026 & - & - & & \\
\hline \multirow[t]{2}{*}{ HDFC Bank Ltd } & Between groups & 0.120 & 11 & 0.011 & 0.946 & 0.500 & \multirow[t]{2}{*}{0.088} & \multirow[t]{2}{*}{-0.005} \\
\hline & Within groups & 1.245 & 108 & 0.012 & - & - & & \\
\hline \multirow[t]{2}{*}{ Hero Honda Motors Ltd } & Between groups & 0.154 & 11 & 0.014 & 0.879 & 0.563 & \multirow[t]{2}{*}{0.082} & \multirow[t]{2}{*}{-0.011} \\
\hline & Within groups & 1.722 & 108 & 0.016 & - & - & & \\
\hline \multirow{2}{*}{$\begin{array}{l}\text { Hindustan Petroleum } \\
\text { Corporation Ltd }\end{array}$} & Between groups & 0.093 & 11 & 0.008 & 1.011 & 0.442 & 0.093 & 0.001 \\
\hline & Within groups & 0.899 & 108 & 0.008 & - & - & & \\
\hline ICICI Bank Ltd & Between groups & 0.281 & 11 & 0.026 & 0.613 & 0.814 & 0.054 & -0.037 \\
\hline & Within groups & 4.499 & 108 & 0.042 & - & - & & \\
\hline Infosys Technologies & Between groups & 0.312 & 11 & 0.028 & 1.274 & 0.249 & 0.115 & 0.025 \\
\hline Ltd & Within groups & 2.409 & 108 & 0.022 & - & - & & \\
\hline ITC Ltd & Between groups & 0.291 & 11 & 0.026 & 1.847 & 0.055 & 0.158 & 0.073 \\
\hline & Within groups & 1.546 & 108 & 0.014 & - & - & & \\
\hline M\&M Ltd & Between groups & 0.275 & 11 & 0.025 & 1.046 & 0.412 & 0.096 & 0.004 \\
\hline & Within groups & 2.580 & 108 & 0.024 & - & - & & \\
\hline ONGC Ltd & Between groups & 0.061 & 11 & 0.006 & 0.307 & 0.983 & 0.030 & -0.068 \\
\hline & Within groups & 1.962 & 108 & 0.018 & - & - & & \\
\hline Ranbaxy Laboratories & Between groups & 0.333 & 11 & 0.030 & 1.616 & 0.104 & 0.141 & 0.054 \\
\hline Ltd & Within groups & 2.021 & 108 & 0.019 & - & - & & \\
\hline Reliance Industries Ltd & Between groups & 0.042 & 11 & 0.004 & 0.311 & 0.982 & 0.031 & -0.068 \\
\hline & Within groups & 1.338 & 108 & 0.012 & - & - & & \\
\hline Sathyam Computers & Between groups & 0.410 & 11 & 0.037 & 0.873 & 0.569 & 0.082 & -0.012 \\
\hline Services Ltd & Within groups & 4.613 & 108 & 0.043 & - & - & & \\
\hline Wipro Ltd & Between groups & 0.570 & 11 & 0.052 & 1.150 & 0.331 & 0.105 & 0.014 \\
\hline & Within groups & 4.865 & 108 & 0.045 & - & - & & \\
\hline Sensex & Between groups & 0.070 & 11 & 0.006 & 1.099 & 0.369 & 0.101 & 0.009 \\
\hline & Within groups & 0.625 & 108 & 0.006 & - & - & & \\
\hline
\end{tabular}

Table - 16 ANOVA FOR SEASONALITY NSE MONTHLY

\begin{tabular}{|l|l|c|c|c|c|c|c|c|}
\hline \multicolumn{1}{|c|}{ Companies } & & Sum $^{2}$ & df & Mean & F & sign & $\mathbf{R}^{2}$ & $\begin{array}{c}\text { Adjust } \\
\mathbf{R}^{\mathbf{2}}\end{array}$ \\
\hline ACC Ltd & $\begin{array}{l}\text { Between } \\
\text { groups }\end{array}$ & 0.365 & 11 & 0.033 & 2.008 & 0.034 & 0.170 & 0.085 \\
\cline { 2 - 9 } & Within groups & 1.782 & 108 & 0.017 & - & - & & \\
\hline $\begin{array}{l}\text { Ashok Leyland } \\
\text { Ltd }\end{array}$ & $\begin{array}{l}\text { Between } \\
\text { groups }\end{array}$ & 0.199 & 11 & 0.018 & 0.687 & 0.776 & 0.063 & -0.033 \\
\cline { 2 - 9 } & Within groups & 2.973 & 108 & 0.028 & - & - & & \\
\hline
\end{tabular}


Stock Market Seasonality- Time Varying Volatility In The Emerging Indian Stock Market

\begin{tabular}{|c|c|c|c|c|c|c|c|c|}
\hline \multirow[t]{2}{*}{ Bata India Ltd } & $\begin{array}{l}\text { Between } \\
\text { groups }\end{array}$ & 0.317 & 11 & 0.029 & 1.708 & 0.081 & \multirow[t]{2}{*}{0.148} & \multirow[t]{2}{*}{0.061} \\
\hline & Within groups & 1.821 & 108 & 0.017 & - & - & & \\
\hline \multirow[t]{2}{*}{ Bhell } & $\begin{array}{l}\text { Between } \\
\text { groups }\end{array}$ & 0.162 & 11 & 0.015 & 1.136 & 0.341 & \multirow[t]{2}{*}{0.104} & \multirow[t]{2}{*}{0.012} \\
\hline & Within groups & 1.401 & 108 & 0.013 & - & - & & \\
\hline \multirow[t]{2}{*}{ Cipla Ltd } & $\begin{array}{l}\text { Between } \\
\text { groups }\end{array}$ & 0.418 & 11 & 0.038 & 2.078 & 0.028 & \multirow[t]{2}{*}{0.175} & \multirow[t]{2}{*}{0.091} \\
\hline & Within groups & 1.975 & 108 & 0.018 & - & - & & \\
\hline \multirow[t]{2}{*}{$\begin{array}{l}\text { Grasim } \\
\text { Industries Ltd }\end{array}$} & $\begin{array}{l}\text { Between } \\
\text { groups }\end{array}$ & 0.119 & 11 & 0.011 & 0.711 & 0.726 & \multirow[t]{2}{*}{0.068} & \multirow[t]{2}{*}{-0.027} \\
\hline & Within groups & 1.650 & 108 & 0.015 & - & - & & \\
\hline \multirow[t]{2}{*}{$\begin{array}{l}\text { HDFC Bank } \\
\text { Ltd }\end{array}$} & $\begin{array}{l}\text { Between } \\
\text { groups }\end{array}$ & 0.111 & 11 & 0.010 & 0.661 & 0.772 & \multirow[t]{2}{*}{0.063} & \multirow[t]{2}{*}{-0.033} \\
\hline & Within groups & 1.651 & 108 & 0.015 & - & - & & \\
\hline \multirow[t]{2}{*}{$\begin{array}{l}\text { Hero Honda } \\
\text { Motors Ltd }\end{array}$} & $\begin{array}{l}\text { Between } \\
\text { groups }\end{array}$ & 0.156 & 11 & 0.014 & 1.179 & 0.310 & \multirow[t]{2}{*}{0.107} & \multirow[t]{2}{*}{0.016} \\
\hline & Within groups & 1.302 & 108 & 0.012 & - & - & & \\
\hline \multirow{2}{*}{$\begin{array}{l}\text { Hindustan } \\
\text { Petroleum } \\
\text { Corporation Ltd }\end{array}$} & $\begin{array}{l}\text { Between } \\
\text { groups }\end{array}$ & 0.251 & 11 & 0.023 & 1.888 & 0.049 & \multirow[t]{2}{*}{0.161} & \multirow[t]{2}{*}{0.076} \\
\hline & Within groups & 1.306 & 108 & 0.012 & - & - & & \\
\hline \multirow[t]{2}{*}{ ICICI Bank Ltd } & $\begin{array}{l}\text { Between } \\
\text { groups }\end{array}$ & 0.260 & 11 & 0.024 & 1.075 & 0.388 & \multirow[t]{2}{*}{0.099} & \multirow[t]{2}{*}{0.007} \\
\hline & Within groups & 2.378 & 108 & 0.022 & - & - & & \\
\hline \multirow{2}{*}{$\begin{array}{l}\text { Infosys } \\
\text { Technologies } \\
\text { Ltd } \\
\end{array}$} & $\begin{array}{l}\text { Between } \\
\text { groups }\end{array}$ & 0.209 & 11 & 0.019 & 1.116 & 0.356 & \multirow[t]{2}{*}{0.102} & \multirow[t]{2}{*}{0.011} \\
\hline & Within groups & 1.838 & 108 & 0.017 & - & - & & \\
\hline \multirow[t]{2}{*}{ ITC Ltd } & $\begin{array}{l}\begin{array}{l}\text { Between } \\
\text { groups }\end{array} \\
\end{array}$ & 0.214 & 11 & 0.019 & 1.639 & 0.098 & 0.143 & 0.056 \\
\hline & Within groups & 1.282 & 108 & 0.012 & - & - & & \\
\hline M\&M Ltd & $\begin{array}{l}\text { Between } \\
\text { groups }\end{array}$ & 0.233 & 11 & 0.021 & 1.188 & 0.304 & 0.108 & 0.017 \\
\hline & Within groups & 1.929 & 108 & 0.018 & - & - & & \\
\hline ONGC Ltd & $\begin{array}{l}\text { Between } \\
\text { groups }\end{array}$ & 0.037 & 11 & 0.003 & 0.245 & 0.993 & 0.024 & -0.075 \\
\hline & Within groups & 1.496 & 108 & 0.014 & - & - & & \\
\hline $\begin{array}{l}\text { Ranbaxy } \\
\text { Laboratories }\end{array}$ & $\begin{array}{l}\text { Between } \\
\text { groups }\end{array}$ & 0.167 & 11 & 0.015 & 1.141 & 0.337 & 0.104 & 0.013 \\
\hline Ltd & Within groups & 1.436 & 108 & 0.013 & - & - & & \\
\hline $\begin{array}{l}\text { Reliance } \\
\text { Industries Ltd }\end{array}$ & $\begin{array}{l}\text { Between } \\
\text { groups }\end{array}$ & 0.072 & 11 & 0.007 & 0.625 & 0.804 & 0.060 & -0.036 \\
\hline & Within groups & 1.138 & 108 & 0.011 & - & - & & \\
\hline $\begin{array}{l}\text { Sathyam } \\
\text { Computers }\end{array}$ & $\begin{array}{l}\text { Between } \\
\text { groups }\end{array}$ & 0.192 & 11 & 0.017 & 0.561 & 0.856 & 0.054 & -0.042 \\
\hline Services Ltd & Within groups & 3.353 & 108 & 0.031 & - & - & & \\
\hline Wipro Ltd & $\begin{array}{l}\text { Between } \\
\text { groups }\end{array}$ & 0.642 & 11 & 0.058 & 1.450 & 0.162 & 0.129 & 0.040 \\
\hline & Within groups & 4.349 & 108 & 0.040 & - & - & & \\
\hline Nifty & $\begin{array}{l}\begin{array}{l}\text { Between } \\
\text { groups }\end{array} \\
\end{array}$ & 0.057 & 11 & 0.005 & 1.140 & 0.338 & 0.104 & 0.013 \\
\hline & Within groups & 0.488 & 108 & 0.005 & - & - & & \\
\hline
\end{tabular}

The analysis of variance has been applied to all sample companies listed both at BSE and NSE to test the null hypothesis that the means of the stock return and market return for five days are equal. The calculate values of ANOVA ' $F$ ' is compared to table of ' $F$ ' at 4 degrees of freedom. The calculated ANOVA results are less than the table value (6.39) of ' $F$ '. Hence, it is concluded that the means of the stock return and market return for five days are equal for the sample companies listed at BSE and at NSE. This is applicable to both daily and monthly returns of sample companies. And also it is found that none of the company has unequal mean returns.

\section{Suggestions}

In wide-ranging, Indian financial market is said to be underdeveloped market when compared to the markets of foreign countries. The efficient financial markets lead to efficient functions of the stock exchanges. It 
is here by suggested that steps should be taken by the authority concerned to develop the financial market on the lines of foreign markets. The short-term researches on daily and monthly fluctuations in the company's market return and stock return can be done and the results can be informed to the investors. The will be much helpful to them to take wise investment decision.

The recent past changes in the market and stock returns and companies can be shown by trend lines with the help of an electronic device. This will help to the investors to take quick decision without referring any credentials. The factors that primarily cause for the high volatility should be indicated to the investors. Insidertrading occupies a predominant role in some company. It leads to deceptive activities by the companies. Rules and regulation in issuing shares should be strictly modified to protect the investors and to avoid artificial increase and decrease in the prices of shares of companies. Stable profits of companies will lead to stability in the market returns and stock returns of companies. Further it minimizes the degree of Preakness of volatility.

There are special rating agencies in India to rate the prices of shares and securities of companies listed at BSE and NSE. But they use different symbols and terms to indicate the extent of risk that associated with the companies. It is suggested that these rating agencies have to take on uniform methodology and avoid intricate terms in order to make possible recognition of market risks. Stability of Government and stability of Governments policies are very important. They affect the market return and stock return. Therefore care should be taken while framing Governments policies in taking sides and cost-effective affairs. Policies like export import policy, fiscal policy and other important policies should be taken without affecting the stock markets with national interests. It is suggested that the number of companies can be included both at BSE and NSE. It will give a inclusive views and ideas on the market return and stock return of the listed companies. It will be very helpful to the investor if they are given reasons for changes in the previous market return and stock return. It will help them to take correct decision in future investment.

\section{Conclusion}

The role of industry in monetary development of a country is very important. It is said to be a country is developed country when it has well industrial development and its share in the country's national output is very significant. The industrial development is necessary to develop the other sectors of an economy since they are mutually interrelated each other. As far as developing countries like India is concerned; there is lack of industrial growth for desire of capital resources. In those days the business enterprises raised their money only by way of financial institutions and on their own. In a course of time, the role of peoples' saving started to enter into the industrial finance in the forms of shares and stocks. There is need foe existence of stock exchanges in it to acquire the savings of the people and lend them to the industrial houses which they need.

But, there is existence of greater degree of risk associated with this investment that there is scope for big gain or big loss. In order to have gain, the investors carefully watch the profitability a solvency of the business firms. In this situation the volatility and seasonality of market return and stock return of the companies occupy an important place. The Bombay Stock Exchange and National Stock Exchange play crucial roles in preparing the volatility and seasonality of the returns on shares of companies. The high volatility leads to ambiguity in the gain and low volatility leads to conviction of gain. The present study analyzed the volatility and seasonality of market return and stock return of some selecte companies in India listed at Bombay Stock Exchange and National Stock Exchange. From this analysis there have been ups and downs in the stability of returns on the share and stocks. The present study concludes that the volatility and seasonality of market return and stock return of sample companies are not same in all the days of a week, in all the months of the year. This is due to changes in the socio, economic and political factors within the country and outside the country. If the above suggestions are fulfilled, the stability in the stock return and market return can be normalized and it will bring more funds to the company and more gains to the investor ultimately India will flourished in the economic development.

\section{References:}

[1]. Akgiray, v. 1989. Conditional heteroskedasticity in time series of stock returns: evidence and forecasts, journal of business 62(1).

[2]. Alina Lucia, trifan. (2009) testing capital asset pricing model for Romanian capital market. In: annals university apulensis series economical. Repec:alu:journl:v:1:y:2009

[3]. Allen, Franklin, and Douglas gale, 1994, limited market participation and volatility of asset prices, American economic review84,

[4]. .Amanulla, S., M.Thiripalraiu, "Week End Effect: New Evidence from the Indian Stock Market". Vikalpa, 26(2), pp. 33 -50, 2001.

[5]. Amihud, Y., Christensen, B.J. And Mendelssohn, h. (1993). Further evidence on the risk-return relationship, working paper, New York University.

[6]. Amin, k., and v. $\mathrm{Ng}, 1997$, "inferring future volatility from the information in implied volatility in Eurodollar options: a new approach", review of financial studies, 10 , pp.

[7]. An empirical investigation of the persistence of stock and bond return seasonality. By: Lavin, Angeline m., journal of applied business research, 08927626, spring2000,

[8]. An empirical investigation of the persistence of stock and bond return seasonality. By: Lavin, Angeline m., journal of applied business research, 08927626, spring2000, 
[9]. Bruce, Burton. (2005) concurrent capital expenditure and the stock market reaction to corporate alliance announcements. In: applied financial economics..

[10]. Barrett, William P Stock markets are scary enough.., Forbes, 00156914, 12/24/2007, vol. 180, issue 13

[11]. Calpers: 'reality check'. By: chernoff, Joel, pensions \& investments, 10504974, 7/11/2005, vol. 33, issue 14

[12]. Campbell, john y., 1987, stock returns and the term structure, journal of financial economics 18.

[13]. Campbell, john y., and john h. Cochrane, 1999, force of habit: a consumption-based explanation of aggregate stock market behavior, journal of political economy107,

[14]. Canina, 1., and s. Figlewski, 1993, "the information content of implied volatility," review of financial studies, 6.

[15]. Carl, Chen; peter, lung; f., Wang. (2009) mispricing and the cross-section of stock returns. In: review of quantitative finance and accounting.

[16]. Chambers, d. R., and s. K. Nawalkha, 2001, "an improved approach to computing implied volatility," financial review, 38.

[17]. Chotigcat, T. Pandey, I.M, "Seasonality in Asia's Emerging Stock Markets: India and Malaysia". International Trade and Finance Association 15th International conference, 2005.

[18]. D and o market troubles not likely to end soon. By: colwell, Megan g., business insurance, 00076864, 9/15/2003, vol. 37, issue 37

[19]. Dan ling, Jiang. (2006) investor overreaction, cross-sectional dispersion of firm valuations, and expected stock returns. In: Ohio state university, Charles a. Dice center for research in financial economics / working paper series.

[20]. Federico, marinelli. (2008) persistence of outstanding performance and shareholder value among diversified firms: the impact of past performance, efficient internal capital market, and relatedness of business segments

[21]. Florian, bardong ; pradeep k., yadav ; söhnke, bartram. (2008) informed trading, information asymmetry and pricing of information risk: empirical evidence from thenyse.

[22]. Guangjie, li. (2009) the horizon effect of stock return predictability and model uncertainty on portfolio choice: uk evidence. In: economics section, Cardiff business school, Cardiff University / Cardiff economics working papers. Repec: cdf: wpaper.

[23]. Hansen, Charlotte Strunk, Accounting \& Finance The relation between implied and realised volatility in the Danish option and equity markets, 08105391, Nov2001, Vol. 41, Issue .

[24]. Harper, Richard B., Asset Allocation, Decoupling, and the Opportunity Cost of Cash. Journal of Portfolio Management, 00954918, Summer2003, Vol. 29, Issue 4

[25]. Hareesh Kumar. Malabika Deo, "Efficiency of Indian Stock Market: A Case of Day of the Week Effect”. SMART Journal of Business Management Studies, 3(2), pp. 28-35, 2007.

[26]. Halperin, Alex China: Is the Reward Worth the Risk?, Business Week Online, 00077135, 4/23/2000.

[27]. Lavin, Angeline M., An Empirical Investigation Of The Persistence Of Stock And Bond Return Seasonality. Journal of Applied Business Research, 08927626, Spring2000, Vol. 16, Issue 2

[28]. Leonid, kogan ; Jiao, Gomes ; motohiro, yogo. (2007) durability of output and expected stockreturns. In: national bureau of economic research, inc/ nber working papers.

[29]. Michael, schroder; richard, stehle ; andreas, schrimpf. (2006) evaluating conditional asset pricing models for the german stock market. In: zew - zentrum für europäische wirtschaftsforschung / center for european economic research / zew discussion papers. Repec: zbw:zewdip:5433.

[30]. Israelsen, Craig L., Dissecting the Decline. Financial Planning, 07467915, Oct2002, Vol. 32, Issue 10

[31]. Michael, Steiner; Manuel, ammann. (2008) risk factors for the Swiss stock market. In: Swiss journal of economics and statistics (sjes). Repec: ses: arsjes: 2008-i-1.

[32]. Michael, greenstone; Paul, oyer. (2005) mandated disclosure, stock returns, and the 1964 securities acts amendments. In: national bureau of economic research, inc / nber working papers..

[33]. Michael, verhofen; Manuel, ammann. (2009) the impact of prior performance on therisk-takingofmutualfundmanagers.In:annuals of finance.

[34]. Moses, Lucia, Taking stock of the market. Editor \& Publisher, 0013094X, 8/12/2002, Vol. 135, Issue 2.

[35]. Nageswari, P. Babu, M, “Analysis of Week End Effect in Indian Stock Market”. SMART Journal of Business Management Studies, 7(1), pp. 78-87, 2011.

[36]. Nageswari, P. Selvam, M., "Re-Examination of the Day of the Week Effect on the Indian Stock Market": A Study With Reference to S\&P CNX 500 Index. Management Trends, 8(1), pp. 29-42, 2011

[37]. Stocks: merrill brings some holiday cheer. By: bogoslaw, david, business week online, 00077135, 12/26/2007

[38]. Swee sum, lam; william wee-lian, ang. (2006) globalization and stock market returns. In: global economy journal.

[39]. Stovall, Sam, Seasonal Softness Ahead? Business Week Online, 00077135, 5/7/200

[40]. Taking stock of the market. By: Moses, Lucia, editor \& publisher, 0013094x, 8/12/2002, vol. 135, issue 29.

[41]. Selvarani, M. Leena Jenefa, "Calendar Anomalies in the National Stock Exchange (NSE) indices". The ICFAI Journal of Applied Finance, 15(1), pp. 56-67, 2009.

[42]. The external view. holland, Andrew, dwor -frecaut, Dominique, jha, sailesh, asia money, 09589309 , nov2004 invest in India, vol. 15

[43]. Weinberg, Neil, The next bubble? : Forbes, 00156914, 12/24/2007, vol. 180, issue 13.

[44]. Unsworth, Edwin Stock market volatility triggering concerns over insurers' investments. , Business Insurance, 00076864, 9/30/2002, Vol. 36, Issue 39

[45]. Ushad Subadar Agathee, "Calendar Effects and the Months of the Year": Evidence from the Mauritian Stock Exchange. International Research journal of Finance and Economics, 14, pp. 254-261, 2008. 